\title{
Development of experimental techniques for the characterization of ultrashort photon pulses of extreme ultraviolet free-electron lasers
}

\author{
S. Düsterer, ${ }^{1}$ M. Rehders, ${ }^{2}$ A. Al-Shemmary, ${ }^{1}$ C. Behrens, ${ }^{1}$ G. Brenner, ${ }^{1}$ O. Brovko, ${ }^{3}$ \\ M. DellAngela, ${ }^{2,4}$ M. Drescher, ${ }^{2}$ B. Faatz, ${ }^{1}$ J. Feldhaus, ${ }^{1}$ U. Frühling, ${ }^{2}$ N. Gerasimova, ${ }^{1,5}$ \\ N. Gerken, ${ }^{2}$ C. Gerth, ${ }^{1}$ T. Golz, ${ }^{1}$ A. Grebentsov, ${ }^{3}$ E. Hass,${ }^{1,2}$ K. Honkavaara, ${ }^{1}$ V. Kocharian, ${ }^{1}$ \\ M. Kurka, ${ }^{6}$ Th. Limberg, ${ }^{1}$ R. Mitzner, ${ }^{7,8}$ R. Moshammer, ${ }^{6}$ E. Plönjes, ${ }^{1}$ M. Richter, ${ }^{9}$ \\ J. Rönsch-Schulenburg, ${ }^{2}$ A. Rudenko, ${ }^{10}$ H. Schlarb, ${ }^{1}$ B. Schmidt, ${ }^{1}$ A. Senftleben, ${ }^{6,11}$ \\ E. A. Schneidmiller, ${ }^{1}$ B. Siemer, ${ }^{7}$ F. Sorgenfrei, ${ }^{2,8}$ A. A. Sorokin, ${ }^{1,12}$ N. Stojanovic, ${ }^{1}$ K. Tiedtke, ${ }^{1}$ \\ R. Treusch, ${ }^{1}$ M. Vogt, ${ }^{1}$ M. Wieland, ${ }^{2}$ W. Wurth, ${ }^{1,2}$ S. Wesch, ${ }^{1,8}$ M. Yan, ${ }^{1}$ M. V. Yurkov, ${ }^{1}{ }^{1}$ \\ H. Zacharias, ${ }^{7}$ and S. Schreiber ${ }^{1}$ \\ ${ }^{1}$ Deutsches Elektronen-Synchrotron, Notkestrasse 85, D-22603 Hamburg, Germany \\ ${ }^{2}$ Universität Hamburg, Physics Department and Center for Free-Electron Laser Science, \\ Luruper Chaussee 149, D-22761 Hamburg, Germany \\ ${ }^{3}$ JINR, Joliot-Curie 6, 141980 Dubna, Moscow region, Russia \\ ${ }^{4}$ FERMI, Elettra - Sincrotrone Trieste, 34149 Basovizza, Trieste, Italy \\ ${ }^{5}$ European XFEL GmbH, Albert-Einstein-Ring 19, 22761 Hamburg, Germany \\ ${ }^{6}$ Max-Planck-Institut für Kernphysik, 69117 Heidelberg, Germany \\ ${ }^{7}$ Physikalisches Institut, Westfälische Wilhelms-Universität, \\ Wilhelm Klemm Strasse 10, 48149 Münster, Germany \\ ${ }^{8}$ Helmholtz-Zentrum Berlin für Materialien und Energie, \\ Albert-Einstein-Strasse 15, 12489 Berlin, Germany \\ ${ }^{9}$ Physikalisch-Technische Bundesanstalt, 12489 Berlin, Germany \\ ${ }^{10}$ J. R. MacDonald Laboratory, Kansas State University, \\ 116 Cardwell Hall, Manhattan, Kansas 66506, USA \\ ${ }^{11}$ Universität Kassel, Institut für Physik, Heinrich-Plett-Strasse 40, 34132 Kassel, Germany \\ ${ }^{12}$ Ioffe Physico-Technical Institute, Polytekhnicheskaya 26, 194021 St. Petersburg, Russia \\ (Received 16 July 2014; published 10 December 2014)
}

\begin{abstract}
One of the most challenging tasks for extreme ultraviolet, soft and hard x-ray free-electron laser photon diagnostics is the precise determination of the photon pulse duration, which is typically in the sub $100 \mathrm{fs}$ range. Nine different methods, able to determine such ultrashort photon pulse durations, were compared experimentally at FLASH, the self-amplified spontaneous emission free-electron laser at DESY in Hamburg, in order to identify advantages and disadvantages of different methods. Radiation pulses at a wavelength of 13.5 and $24.0 \mathrm{~nm}$ together with the corresponding electron bunch duration were measured by indirect methods like analyzing spectral correlations, statistical fluctuations, and energy modulations of the electron bunch and also by direct methods like autocorrelation techniques, terahertz streaking, or reflectivity changes of solid state samples. In this paper, we present a comprehensive overview of the various techniques and a comparison of the individual experimental results. The information gained is of utmost importance for the future development of reliable pulse duration monitors indispensable for successful experiments with ultrashort extreme ultraviolet pulses.
\end{abstract}

DOI: $10.1103 /$ PhysRevSTAB.17.120702

PACS numbers: 41.60.Cr, 41.50.+h

\section{INTRODUCTION}

One of the essential characteristics of the new generation of extreme ultraviolet (XUV) to x-ray free-electron lasers is their ultrashort pulse duration in the femtosecond range. This opens up new opportunities for the detailed

Published by the American Physical Society under the terms of the Creative Commons Attribution 3.0 License. Further distribution of this work must maintain attribution to the author(s) and the published article's title, journal citation, and DOI. investigation of ultrafast reaction dynamics on the femtosecond time scale [1-3], allows novel approaches in structure determination of bio molecules [4], and allows the investigation of multiphoton processes in the XUV [5] to $\mathrm{x}$-ray range [6] which has not been possible before. The accurate knowledge of the free-electron laser (FEL) key parameters such as pulse peak power, radiance, and ontarget irradiance, for example, is crucial for the analysis of experimental data, particularly for nonlinear interactions [7]. It turns out that the number of photons, the focal spot size, and the spectral content in such short pulses can be 
measured reliably [8-11], while the pulse duration is the most difficult parameter to be determined.

FLASH [12] provides a wide range of wavelengths from 4.2 up to $45 \mathrm{~nm}$. With the last upgrade (2010) of the accelerator [13], the duration of the generated photon pulses can be varied between a few tens of femtoseconds up to several hundred femtosecond duration. However, a reliable method to measure pulse durations for the entire parameter range is not yet available. Although a variety of methods have been proposed, they all need to be set up and tested experimentally to find out the most universal, most reliable, and most easy to use technique. In this paper, we present nine different techniques to determine the photon pulse duration. They are either performed in a direct way by measuring the photon pulse duration at the experimental end stations or, on the other hand, by indirect methods measuring only parameters which are linked-by theoretical models-to the actual pulse duration. From the measured information, the actual XUV pulse duration can be calculated by using these models. From the experimental point of view, indirect methods are typically simpler to realize as compared to the direct approaches. However, they have to be verified and calibrated by direct methods initially.

Several individual photon pulse duration measurement campaigns have been undertaken at FLASH in the last years [14-18]. In addition, there was one study comparing electron bunch duration measurements with the result of one indirect photon pulse duration determination technique [19]. However, up to now there were no studies at FLASH or at any other XUV or x-ray FEL where many different methods were compared within one dedicated pulse duration measurement campaign (see Fig. 1).

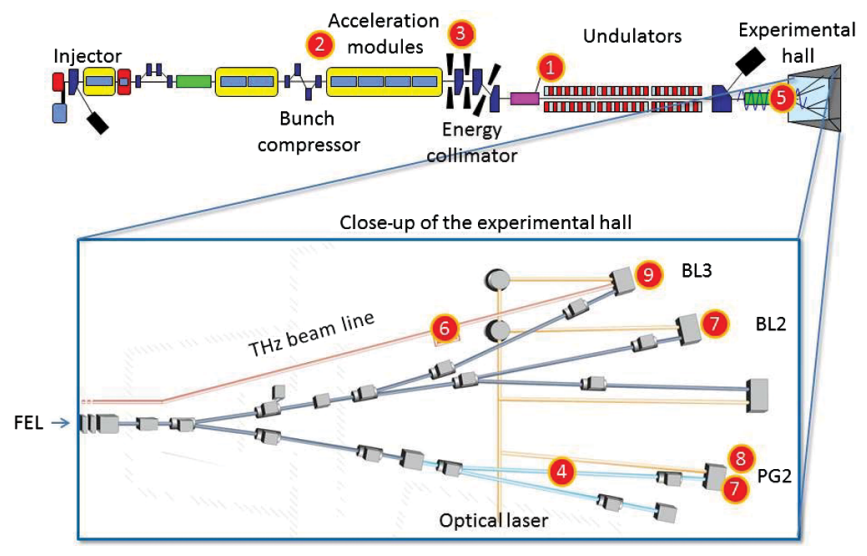

FIG. 1. Schematic layout of the FLASH accelerator and the experimental hall (not to scale). The total length of the facility is $315 \mathrm{~m}$. The positions of the various techniques for electron bunch or photon pulse duration measurements are indicated by numbers: (1) Transverse deflecting rf structure, (2) bunch compression monitors, (3) terahertz spectrometer CRISP, (4) XUV spectra, (5) XUV statistics, (6) optical replica (afterburner), (7) XUV autocorrelation (gas phase), (8) XUV autocorrelation (solid state), and (9) terahertz streaking.
The main motivation for this study was threefold. First, we wanted to address the question to what degree we can correlate the results measured by the indirect methods to the direct ones. What are the uncertainties when comparing the different methods? How much information about the photon pulse duration can we deduce from the electron beam parameters in contrast to the photon-based indirect or direct methods? Second, the realization of all nine techniques together under the same beam conditions allows a direct comparison of advantages and disadvantages of the individual techniques. Third, the aim of the campaign was to identify sensitive parameters of the electron bunch compression and to develop recipes for routine operation to reliably establish a specific user-requested XUV pulse duration at FLASH, especially for ultrashort pulses below $50 \mathrm{fs}$.

\section{ELECTRON BEAM PROPERTIES AND SASE PROCESS}

The amplification process in the self-amplified spontaneous emission free-electron laser (SASE FEL) starts from shot noise in the electron beam, passes a stage of exponential amplification, and finally enters the saturation regime (see Fig. 2). An estimation of the SASE FEL characteristics is frequently performed in the framework of a onedimensional model in terms of the FEL parameter $\rho$ [20]:

$$
\rho=\frac{\lambda_{w}}{4 \pi}\left[\frac{4 \pi^{2} j_{0} K^{2} A_{J J}^{2}}{I_{A} \lambda_{w} \gamma^{3}}\right]^{1 / 3},
$$

and the number of cooperating electrons $N_{c}=I /(e \rho \omega)$, where $\omega$ is the frequency of the electromagnetic (XUV) wave. Here $\gamma$ is the relativistic factor, $j_{0}=I /\left(2 \pi \sigma^{2}\right)$ is the

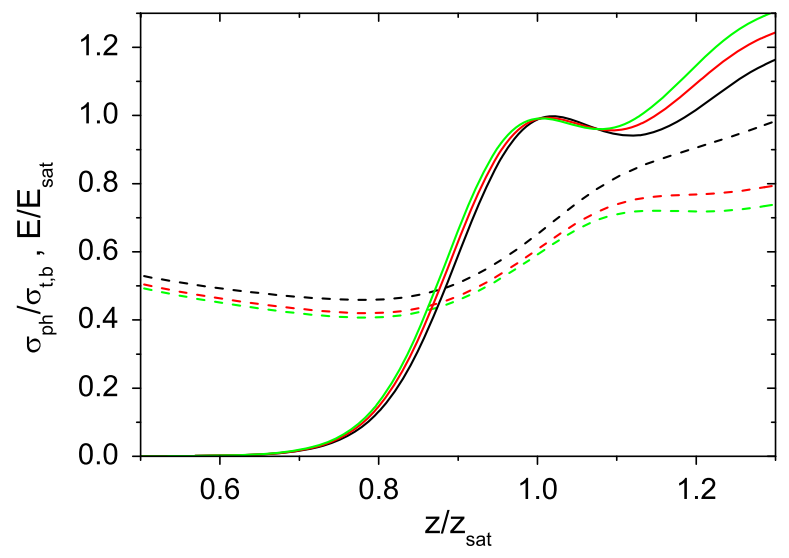

FIG. 2. The evolution of the energy in the radiation pulse $E$ (solid line) and rms photon pulse duration $\sigma_{\mathrm{ph}}$ (dashed line) are shown as functions of the undulator length. The three color codes (black, red, and green) refer to different values of the electron pulse duration $\rho \omega \sigma_{t, b}=2,4$, and 8 . The values are normalized as $E / E_{\mathrm{sat}}$ and $\sigma_{\mathrm{ph}} / \sigma_{t, b}$. For the presented experiments, $\rho \omega \sigma_{t, b}$ was about 4 . 
beam current density, $\sigma=\sqrt{\beta \epsilon_{n} / \gamma}$ is the rms transverse size of the electron beam, $\beta$ is the external focusing beta function, $I_{A}=m c^{3} / e \simeq 17 \mathrm{kA}$ is Alfven's current, $\lambda_{w}$ is the undulator period, and $K$ is the rms undulator parameter. The factors $A_{J J}=1$ and $A_{J J}=\left[J_{0}(Q)-J_{1}(Q)\right]$ describe a helical and a planar undulator, respectively, $J_{n}(Q)$ is a Bessel function of $n$th order, and $Q=K^{2} /\left[2\left(1+K^{2}\right)\right]$. For the field gain length $L_{g}$, the saturation length $L_{\text {sat }}$, and the coherence time at saturation $\tau_{c}^{\text {sat }}$, we have [21-23]

$$
L_{g} \sim \frac{\lambda_{w}}{4 \pi \rho}, \quad L_{\mathrm{sat}} \sim \frac{\lambda_{w}}{4 \pi \rho}\left[3+\frac{\ln N_{c}}{\sqrt{3}}\right], \quad \tau_{c}^{\mathrm{sat}} \simeq \frac{1}{\rho \omega} \sqrt{\frac{\pi \ln N_{c}}{18}} .
$$

To be specific, we consider a Gaussian axial distribution of the electron beam current:

$$
I(t)=I \exp \left(-t^{2} / 2 \sigma_{t, b}^{2}\right)
$$

with an rms electron pulse duration $\sigma_{t, b}$.

These simple physical considerations are confirmed with the results of numerical simulations with the time-dependent simulation code FAST [24]. We consider a model with a Gaussian longitudinal profile of the electron bunch and trace the FEL parameters for different values of the electron rms pulse duration $\rho \omega \sigma_{t, b}$ (a more detailed start-to-end simulation will be described in Sec. VIB). Figure 2 shows the evolution along the undulator of the radiation pulse energy and the rms photon pulse length normalized to the electron pulse duration. During the exponential stage of amplification, the central part of the radiation pulse (due to the higher electron beam current) has a shorter gain length as compared to the tails. This leads to a narrowing of the XUV pulse duration with respect to the electron pulse. At the end of the linear regime, the photon pulse is only 0.4 times the electron pulse length. With increasing propagation length, the XUV pulse duration is increasing again, reaching $~ 0.6$ times the electron pulse duration at saturation. Finally, in deep saturation the photon pulse length grows even more due to slippage effects as illustrated in Fig. 2.

Looking in more detail to the temporal profile of the XUV photon pulses from the SASE amplification process, it turns out that it consists of several spikes (wave packets). These spikes are often denoted as longitudinal modes. The duration of a single spike corresponds roughly to the coherence time, the time over which a correlation in the radiation field is present [25-27]. The radiation pulse energy in the SASE FEL fluctuates from shot to shot due to its origin from shot noise in the electron beam. The radiation from a SASE FEL operating in the linear regime exhibits the properties of completely chaotically polarized light $[22,23]$. One consequence is that the probability distribution of the energy in a radiation pulse fits to a gamma distribution:

$$
p(E)=\frac{M^{M}}{\Gamma(M)}\left(\frac{E}{\langle E\rangle}\right)^{M-1} \frac{1}{\langle E\rangle} \exp \left(-M \frac{E}{\langle E\rangle}\right)
$$

where $\Gamma(M)$ is the gamma function, $M=1 / \sigma_{E}^{2}$, and $\sigma_{E}^{2}=\left\langle(E-\langle E\rangle)^{2}\right\rangle /\langle E\rangle^{2}$. The parameter $M$ is interpreted as the average number of modes in the radiation pulse. The connection between basic FEL parameters given by Eq. (2) and fundamental statistical energy fluctuations given in Eq. (4) reveal a rather simple way to derive the coherence time and the minimum photon pulse duration by the measurement of the saturation length and number of radiation modes [19]:

$$
\tau_{c} \simeq \frac{\lambda L_{\mathrm{sat}}}{2 \sqrt{\pi} c \lambda_{w}}, \quad \sigma_{\mathrm{ph}}^{\min } \simeq \frac{\sigma_{t, b}}{2} \simeq \frac{M \lambda}{10 \rho} \simeq \frac{M \lambda L_{\mathrm{sat}}}{10 c \lambda_{w}} .
$$

The minimum radiation pulse duration can thus be expressed in terms of the coherence time [19,28]:

$$
\sigma_{\mathrm{ph}}^{\min } \simeq 0.35 \times M \times \tau_{c} .
$$

Note that $\sigma_{\mathrm{ph}}^{\min }$ is given in rms while the coherence time $\tau_{c}$ is FWHM. As shown in Fig. 2, the minimum pulse duration is achieved at $80 \%$ of the saturation length, while the actual XUV pulse duration at saturation is $\sim 1.4$ times longer.

\section{A. Electron beam properties and compression}

The superconducting linear accelerator of FLASH operates in a burst mode. It allows the acceleration of bursts of several hundred bunches within a $0.8 \mathrm{~ms}$ long bunch train with a repetition rate of 10 trains per second $(10 \mathrm{~Hz})$. For a more complete description of FLASH, the reader is referred to Refs. [12,29], and references therein.

The electron source is a laser-driven radio frequency (rf) gun photoinjector. A 1.5-cell normal conducting $1.3 \mathrm{GHz}$ $L$-band copper cavity is powered by a 5 MW klystron, pulsed with $10 \mathrm{~Hz}$, yielding a radio frequency pulse duration of up to $830 \mu \mathrm{s}$ [30]. The photocathode is a thin film of $\mathrm{Cs}_{2} \mathrm{Te}$ deposited on a molybdenum plug, inserted into the rf gun backplane via a load-lock system [31]. The injector laser is based on a mode-locked bunch train oscillator synchronized to the $1.3 \mathrm{GHz}$ rf of the accelerator. A chain of diode-pumped Nd:YLF amplifiers provides enough power to convert the initial infrared wavelength into ultraviolet $(262 \mathrm{~nm})$ pulses [32].

The electron bunch charge is variable to a certain extent: a charge between $80 \mathrm{pC}$ and $1 \mathrm{nC}$ is used during FEL operation. The number of electron bunches per bunch train can be varied from a single bunch up to 2400 . Since the bunch train length is limited to $800 \mu \mathrm{s}$, the maximum number of bunches in a train depends on the spacing between the bunches. Several distinct spacings corresponding to frequencies between $3 \mathrm{MHz}$ and $40 \mathrm{kHz}$ are possible.

FLASH uses seven superconducting accelerating modules to boost the electron energy up to $1.25 \mathrm{GeV}$. Each 
module consists of eight nine-cell standing wave niobium cavities with a fundamental mode frequency of $1.3 \mathrm{GHz}$. The cavities are bath cooled by superfluid helium to $2 \mathrm{~K}$. The length of each accelerating module is $12.2 \mathrm{~m}$ [12].

Two magnetic chicane bunch compressors are used to shorten the electron bunches to a level of a few tens to a few hundreds of femtoseconds. The first compression occurs at $150 \mathrm{MeV}$ electron energy, while the second compression stage is at $450 \mathrm{MeV}$. An almost linear energy chirp is imposed onto the bunch. Compression is achieved, when the head of the bunch travels a longer path through the chicane than the tail. The appropriate energy chirp is achieved by accelerating off crest in an accelerating structure before the chicane. Since the accelerating field has a cosine shape, the energy chirp is not exactly linear. Four cavities operating at the third-harmonic rf frequency $(3.9 \mathrm{GHz})$ are used to flatten the energy chirp before the first bunch compressor. With the correct combination of off-crest phase in the $1.3 \mathrm{GHz}$ cavities and the phase and power of the $3.9 \mathrm{GHz}$ cavities, the energy chirp can be linearized and the compression ratio can be adjusted to a certain extent as well. To mitigate space charge-induced beam instabilities, the bunch charge needs to be adjusted appropriately. In general, the smaller the charge, the stronger the compression can be chosen.

A dedicated low-level rf system stabilizes and flattens the amplitude and phase of the accelerating field in the rf gun and the accelerating modules with feedback and feedforward methods. An excellent rms energy stability of better than $\sigma_{E} / E=10^{-4}$ is achieved; the phase stability is better than $0.01^{\circ}$. Because of the bunch compressor chicanes, this energy jitter is translated into an arrival time rms jitter of $60 \mathrm{fs}$ of the electron bunches in respect to the master rf. An optical synchronization system based on the bunch arrival time detection is being set up at FLASH with the goal to stabilize the arrival time of all bunches in the bunch train to a $10 \mathrm{fs}$ level [33]. This is important for proper acceleration of long bunch trains, for seeding schemes with external lasers, and for pump-probe experiments.

\section{ELECTRON BUNCH DURATION MEASUREMENTS}

\section{A. Time domain measurements: The transverse deflecting rf structure}

A transverse deflecting rf structure (TDS) provides a direct single-shot method to measure the longitudinal phase space of an electron bunch with femtosecond resolution [34,35]. At FLASH, an $S$-band TDS structure called LOLA originally built at SLAC for a different purpose $[36,37]$ is installed just upstream from the SASE undulators in combination with a magnetic energy spectrometer. At the $\mathrm{x}$-ray free-electron laser LCLS, such a device was recently installed downstream from the undulators, which allows one to actually determine the lasing part of the electron bunch, and thus this device can measure the x-ray pulse duration [38]. In the FLASH case, the TDS measures only the electron bunch properties.

The operating principle is similar to that of a streak camera. The rf field of the structure deflects the electron beam vertically. The longitudinal bunch shape is transferred into the transverse plane, which is measured with a suitable screen and camera system. Meanwhile, two options are available.

First, a fast kicker magnet is used to select an arbitrary bunch out of the bunch train. The bunch to be analyzed is kicked on an off-axis screen in the straight section, providing a measurement of the longitudinal profile. All other bunches proceed on their usual path straight to the beam dump.

Second, the first bunch of the train is streaked and then sent to an energy spectrometer laid out in the horizontal plane, perpendicular to the streak direction. This method provides a single-shot measurement of the longitudinal phase space, time, and energy. In this mode, only two bunches in the train are allowed, and the selection of an arbitrary pulse is not possible, since the spectrometer line does not provide a suitable beam dump. Details on the measurement principles, the experimental setup, and technical descriptions can be found in Refs. [19,39,40]. During the presented experiments, the first option was not yet implemented, and the TDS was operated only with one or maximum two bunches in a bunch train.

For an electron passing through a vertically deflecting TDS around the zero crossing of the rf phase at a position $s_{0}$, the vertical offset of the electron at a downstream position $s$ is given by [19,39]

$$
y_{ \pm}(s)=y_{0}(s)+C_{y}\left(s, s_{0}\right) z / c \pm S_{y}\left(s, s_{0}\right) z / c,
$$

where $C_{y}\left(s, s_{0}\right)$ is the intrinsic longitudinal-to-vertical linear correlation, $y_{0}$ is the intrinsic offset, $c$ is the speed of light in vacuum, and $z$ is the longitudinal position of the electron relative to the zero crossing of the rf phase. $S_{y}\left(s, s_{0}\right)$ is the shear parameter, which is a measure of the kick strength of the TDS, at positive and negative rf phase zero crossings, respectively. This equation describes a linear transformation of the longitudinal coordinate to the vertical coordinate. For consistency in this paper, the longitudinal coordinate will be expressed in the time domain as $t=-z / c$.

The vertical rms beam size $\sigma_{y}$ of a sheared bunch at $s$ is then

$$
\sigma_{y_{ \pm}}^{2}(s)=\sigma_{y_{0}}^{2}+\left[C_{y}\left(s, s_{0}\right) \pm S_{y}\left(s, s_{0}\right)\right]^{2} \sigma_{t, b}^{2} .
$$

By measuring the vertical $\mathrm{rms}$ beam sizes at a positive and negative shear parameter $\pm S_{y}$ and for $S_{y}=0$ (TDS switched off), the intrinsic linear correlation $C_{y}$, the intrinsic vertical rms beam size $\sigma_{y_{0}}$, and finally the rms 
bunch duration $\sigma_{t, b}$ are obtained. The rms time resolution is given by $\mathcal{R}_{t, e}^{ \pm}=\sigma_{y_{0}} /\left|C_{y} \pm S_{y}\right|$ (see Ref. [19] for details).

In general, the temporal resolution of measurements with TDS depends on the accelerator optics, beam energy, TDS deflecting voltage, and frequency. With the existing $S$-band TDS $(2.856 \mathrm{GHz})$ at FLASH, a resolution of 5 fs has been obtained for a beam energy of $1 \mathrm{GeV}$. Better resolution can be achieved by utilizing deflecting structures at higher frequencies; e.g., LCLS has reported a temporal resolution of less than $1 \mathrm{fs}$ with the $X$-band structure [38].

In the horizontal plane, the energy spectrometer disperses the electron beam to

$$
x(s)=x_{0}(s)+D_{x}\left(s, s_{0}\right) \delta,
$$

where $D_{x}$ is the horizontal dispersion and $\delta \approx \Delta E / E$ is the energy deviation of the electrons in respect to the mean energy $E$ of the beam. The measurements presented in this paper use the TDS in combination with the spectrometer and for the first bunch only. The streaked beam shape is measured by using a $100 \mu \mathrm{m}$ thick Ce:YAG scintillator screen imaged on a CCD camera.

Figure 3 shows two examples of single-shot measurements of the longitudinal phase space. FLASH has been set up for lasing at 13.5 and $24 \mathrm{~nm}$ with a bunch charge of $0.15 \mathrm{nC}$. From the projection on the time axis we obtain a peak current of 2.5 and $1.5 \mathrm{kA}$, respectively. In both cases, the leading part of the bunches (the right-hand side of the
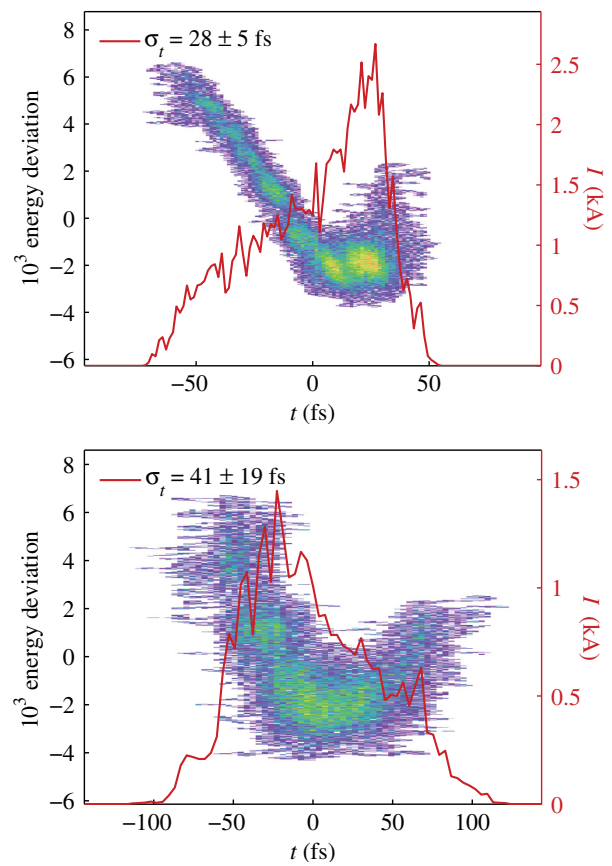

FIG. 3. Single-shot longitudinal phase space measurements for the two runs at 13.5 (upper figure) and $24 \mathrm{~nm}$ (lower figure). The rms bunch lengths shown are calculated without corrections of the longitudinal-to-vertical correlation and the intrinsic vertical beam size. The projection onto the time axis gives the current profile of the electron bunch, shown in red. horizontal axis) has a larger energy than the trailing part. The respective rms bunch lengths given in the legend of Fig. 3 are without correction for the intrinsic vertical rms beam size $\sigma_{y_{0}}$ and for a potential longitudinal-to-vertical correlation $C_{y}$. The measurement errors given result from the calibration uncertainty of the shear parameter: $S_{y} / c=(15 \pm 7)$ fs and $S_{y} / c=(38 \pm 7)$ fs, respectively.

To determine the statistical uncertainty and to take into account the corrections for $\sigma_{y_{0}}$ and $C_{y}, 20$ single-shot measurements were performed at each of the three points $\pm S_{y}$ and $S_{y}=0$. As shown in Fig. 4, the corrected average rms bunch durations are determined to be $23 \pm 5$ (for $13.5 \mathrm{~nm}$ ) and $37 \pm 18 \mathrm{fs}$ (for $24 \mathrm{~nm}$ ), respectively. A linear longitudinal-to-vertical correlation $C_{y} / c$ with a strength of about $10 \%$ of the shear parameter was determined for both settings. With the estimation of the intrinsic vertical rms beam size $\sigma_{y_{0}}$, the rms longitudinal time resolution $\mathcal{R}_{t, e}^{ \pm}$can be calculated to be $\mathcal{R}_{t, e}^{+}=6$ fs $\left(\right.$ at $\left.+S_{y}\right)$ and $\mathcal{R}_{t, e}^{+}=15$ fs (at $\left.+S_{y}\right)$, respectively.

\section{B. Frequency-domain measurements}

An alternative method to resolve the longitudinal bunch profile is to measure the coherent terahertz radiation
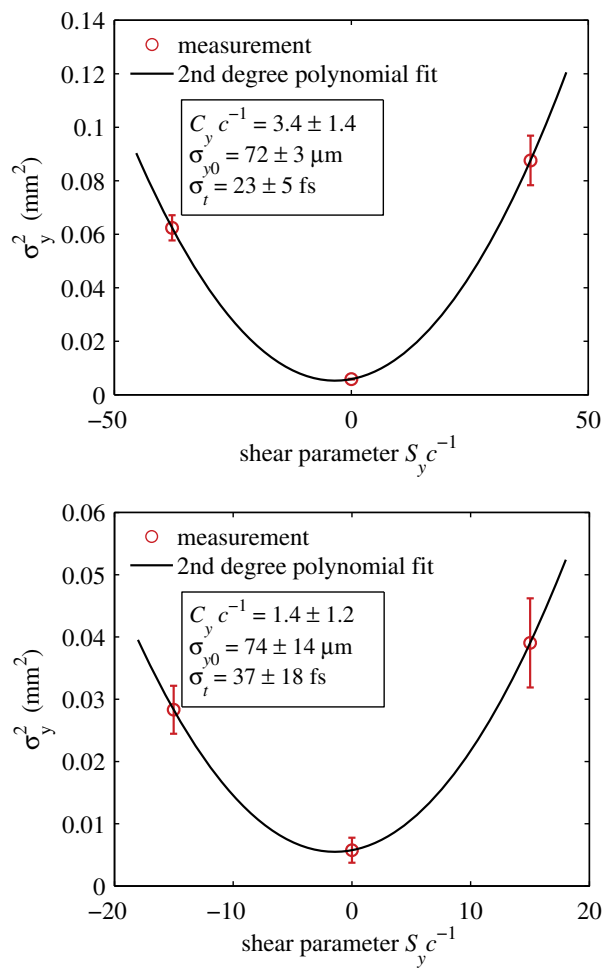

FIG. 4. Calculation of the longitudinal rms bunch length $\sigma_{t, b}$ including statistical errors and corrections due to the intrinsic longitudinal-to-vertical correlation $C_{y}$ and the intrinsic vertical rms beam size $\sigma_{y_{0}}$. The plots show the measured vertical rms beam sizes $\sigma_{y}^{2}$ averaged over 20 single shots for three shear parameters, at $\pm S_{y}$ and $S_{y}=0$. The upper plot data have been taken during the $13.5 \mathrm{~nm}$ run, the lower plot during the $24 \mathrm{~nm}$ run. The black curve represents a 2 nd degree polynomial fit. 
emitted by the electron bunch [coherent transition radiation (CTR)]. For electron bunch structures which are smaller than a specific wavelength, the single electron fields superimpose coherently and the radiated intensity scales quadratically with the number of particles. This increase of spectral energy can be measured. Neglecting the transverse effects for simplicity, the intensity distribution of the coherent radiation emitted by $N$ particles of the electron bunch is given by the equation

$$
\frac{d U_{\mathrm{coh}}}{d \lambda} \approx N^{2} \frac{d U_{1}}{d \lambda}\left|F_{l}(\lambda)\right|^{2}
$$

where $d U_{1} / d \lambda$ is the spectral intensity radiated by a single electron and $F_{l}(\lambda)$ is the complex longitudinal form factor of the bunch. This form factor is the Fourier transform of the longitudinal normalized particle density distribution $\rho_{l}(z)$ :

$$
\begin{aligned}
F_{l}(\lambda) & =\left|F_{l}(\lambda)\right| \exp [i \Phi(\lambda)] \\
& =\int_{-\infty}^{\infty} \rho_{l}(z) \exp (-2 \pi i z / \lambda) \mathrm{d} z .
\end{aligned}
$$

\section{Bunch shape reconstruction by terahertz spectroscopy}

The newly developed coherent radiation intensity spectrometer with four stages (CRISP4), which is located just behind the last accelerator module, measures directly the coherent spectrum of the CTR; see Eq. (10). Out of the FLASH bunch train, one single bunch can be deflected onto an off-axis screen by a fast kicker magnet. The emitted CTR is transported by a beam line to the spectrometer in an external building [41]. The device is capable of operating in parallel with FEL operation.

The spectrometer itself houses two different sets of five consecutive dispersive reflecting gratings and 120 parallel readout channels. It enables one to cover a very large wavelength range simultaneously, from 5 to $44 \mu \mathrm{m}$ and 45 to $440 \mu \mathrm{m}$, respectively. For further technical information, see [42].

Measurement of the absolute intensity of the CTR allows the determination of the absolute value of the form factor $\left|F_{l}\right|$; however, the phase $\Phi(\lambda)$ remains unknown. To determine the phase and to reconstruct the temporal profile of the bunch, the Kramers-Kronig relation is used [43]. This technique allows the retrieval of the minimal phase $\Phi_{\min }(\lambda)$ which determines the most compact bunch shape which fits a given form factor. With the reconstructed phase, the longitudinal profile of the electron bunch can be calculated by using inverse Fourier transformation.

Figure 5 shows the measured form factor with CRISP4 (top) and the reconstructed temporal profile for $13.5 \mathrm{~nm}$ settings. (bottom). Typically, the reconstructed profiles are in excellent agreement with measurements in the time domain using the TDS [44].
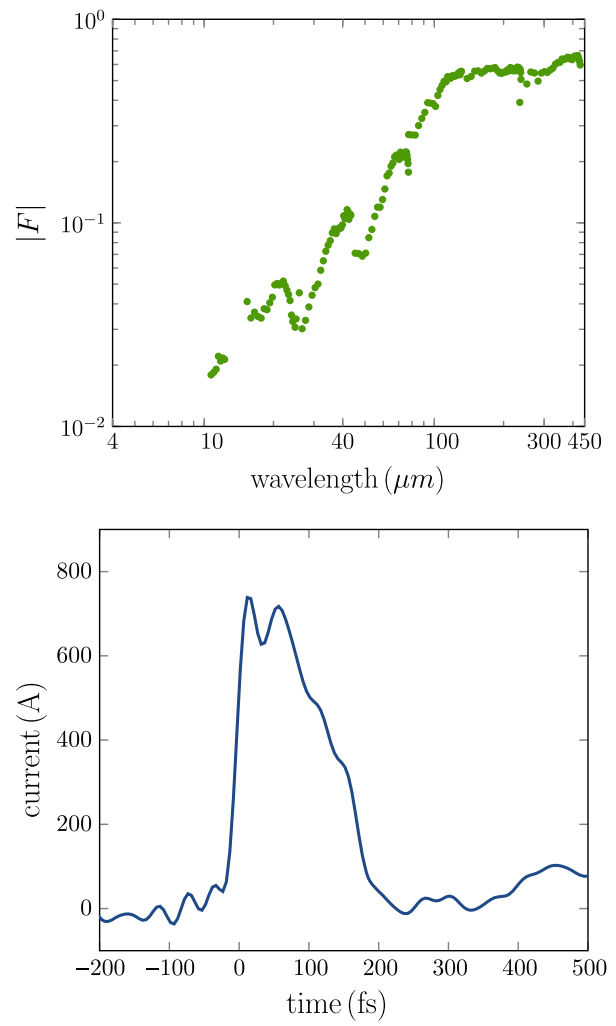

FIG. 5. The upper figure shows the form factor measured by the coherent radiation intensity spectrometer (CRISP4). The lower figure presents the reconstructed temporal profile of the electron bunch for the $13.5 \mathrm{~nm}$ run.

CRISP4 is an easy to use bunch length diagnostic tool for measuring single-shot bunch profiles for a freely selectable bunch in the bunch train.

\section{Bunch compression monitors}

A common practice at FEL facilities for monitoring the bunch length is to measure the spectrally integrated intensity of the emitted coherent radiation over a properly defined range of wavelengths $[45,46]$

$$
I_{\mathrm{coh}}=\int \frac{d U_{\mathrm{coh}}}{d \lambda} \mathrm{d} \lambda
$$

The total intensity increases monotonically with decreasing bunch length. In general, the integration range has to be set to a certain frequency range according to the pulse duration range to be measured. This technique easily allows monitoring of bunch length variations and setting up a compression feedback. For an absolute bunch length determination, the monitor has to be calibrated by independent measurements. For sufficiently short bunch lengths, the total coherent intensity is largely determined by the overall length of the bunch, while for longer bunches, the internal structure of the bunch becomes more and more important. In this case, a unique determination of the bunch length 
from the sole value of the integrated intensity is no longer possible.

The use of noninvasive radiation sources like synchrotron or diffraction radiators allows one to run such a monitor nondestructively on all bunches of the train during normal FEL operation.

At FLASH the bunch compression monitors (BCMs) consist of single pyroelectric elements which detect coherent diffraction radiation when the electron bunches pass the aperture of a slitted screen [47]. The BCMs are located behind the 1st and the 2nd bunch compressors. For measurements shown in this paper, the BCM after the 2nd bunch compressor was used; see Fig. 1.

Developing an elaborate and detailed model of the whole setup and performing comparative measurements with the TDS, the monitor was calibrated to give absolute bunch length data. The usable range of FLASH's BCMs spans from $30 \mathrm{fs}$ rms (saturation due to short wavelength transmission cutoff) to $600 \mathrm{fs}$ rms (loss of uniqueness for different longitudinal profiles). In this regime, the typical uncertainty for the bunch length is about $10 \%$. Further details can be found in Ref. [48].

\section{XUV PULSE DURATION MEASUREMENTS: INDIRECT METHODS}

\section{A. Statistical fluctuations of the radiation energy}

The measurement of the statistical fluctuations of the pulse energy of a SASE FEL allows one to estimate the lasing fraction of the electron pulse length and thus the photon pulse duration. This technique has been effectively used at FLASH and LCLS $[12,19,49,50]$. First, the SASE FEL is tuned to the saturation regime. The knowledge of the saturation length gives us an estimate for the coherence time using Eq. (5). Then the FEL process is stopped at the end of the high gain linear regime (FEL power is reduced by a factor of 20 with respect to the saturation regime), and measurements of the radiation pulse energies were performed. The inverse squared value of the radiation pulse energy fluctuations gives the number of the radiation modes $M$ in the pulse [see Eq. (4)]. Thus, we define all quantities required for the derivation of the radiation pulse duration in the end of the high gain linear regime [see Eq. (5)]. The photon pulse lengthening in the nonlinear regime depends on the undulator length and corresponds to a factor of 1.4 at the saturation point (see Fig. 2). A detailed description of the experimental procedure can be found in Ref. [19].

As an example, the experimental data from the $13.5 \mathrm{~nm}$ run is shown in Figs. 6 and 7. The energy of the radiation pulse was measured with a detector based on a microchannel plate (MCP) $[29,51,52]$. Fluctuations of the electron beam and accelerator parameters may in addition contribute to the fluctuations of the radiation pulse energy. But only fundamental SASE FEL fluctuations are to be
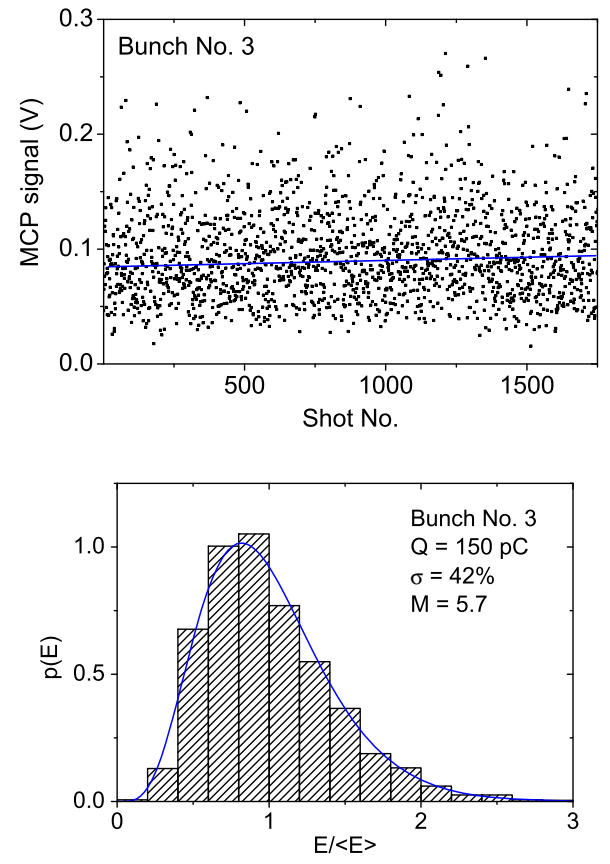

FIG. 6. Experimental data from FLASH operating in the linear regime. The active undulator length is $13.5 \mathrm{~m}$ (three undulator modules). The radiation wavelength is $13.5 \mathrm{~nm}$. Left plot: Raw data of MCP detector for bunch number 3. The solid curve shows the averaged value. Right plot: Probability distribution of the radiation energy for bunch number 3 . The solid curve represents the gamma distribution with $M=5.7$.
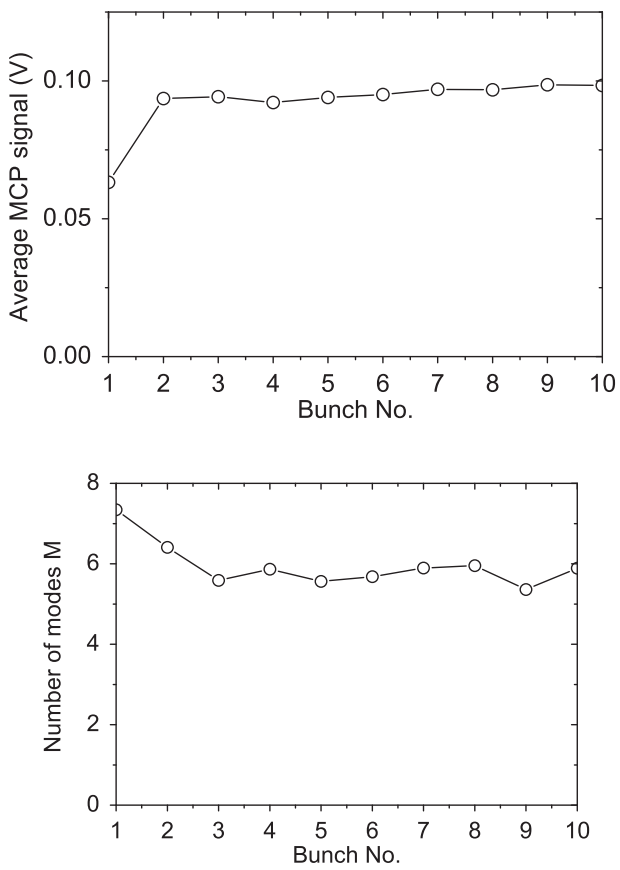

FIG. 7. Experimental data from FLASH operating in the linear regime. Active undulator length is $13.5 \mathrm{~m}$ (three undulator modules). Upper plot: The average value of the MCP signal versus the position of the bunch in the train. Lower plot: Number of modes in the radiation pulse versus the position of the bunch in the train. 
detected for the precise statistical analysis. In addition to the MCP signals, several other accelerator parameters like bunch charge monitor readings, beam position monitor readings, etc., are recorded. If the deviation is larger than a prescribed threshold, this event is excluded from the data set. The upper plot in Fig. 6 shows the raw data of the MCP readings for bunch number 3 in the train versus shot number. The lower plot in this figure shows the probability distribution of the energy in the radiation pulse for bunch number 3. Using the statistical method, we can resolve every bunch in the train. The plots in Fig. 7 show the evolution of the average MCP signal (average energy in the radiation pulse) and of the number of radiation modes along the bunch train. These plots highlight a typical feature of the multibunch operation of FLASH: With optimal tuning, all bunches in the train except of the first one or two pulses show very similar features in terms of intensity and number of modes. Thus, we find an average of $5.8 \pm 0.2$ modes for all bunches (excluding the first bunch). Measurements of the radiation intensity along the undulator (gain curve) yield the gain length, and thus the coherence time can be calculated [Eq. (5)]. In the $13.5 \mathrm{~nm}$ case, the value for the coherence time is about 9 fs (FWHM) [19,53]. Then Eq. (6) can be used to estimate the radiation pulse length. This estimate assumes a high degree of transverse coherence which is true for the parameter space of FLASH [53]. The pulse duration at the end of the linear regime equals $40 \pm 5$ fs (FWHM). The lengthening of the radiation pulse which occurs in the nonlinear regime results finally in a duration of $60 \pm 10 \mathrm{fs}(\mathrm{FWHM})$ at the end of the undulator.

\section{B. Spectral correlations}

As already discussed in Sec. II, the SASE FEL radiation has its source in the initial random fluctuations of the electron beam current density within the electron bunch, and the statistical nature of the electron beam shot noise directly affects the characteristics of the output radiation. The temporal profile of XUV photon pulses originating from the SASE amplification process exhibits subspikes. The width of these intensity peaks is related to the SASE coherence time-the time over which a correlation in the radiation field exists. For short photon pulses, only a few subspikes are observed, while long pulses consist of up to several dozens of spikes. Since the photon spectrum is simply the Fourier transform of the temporal intensity profile, it is expected that in the spectral domain we can also observe a spiky structure. In fact, the spectral intensity profile also consists of spikes, as can be seen in Fig. 8. The width of the spectral peaks is inversely proportional to the electron bunch duration. A detailed description of the relation between the number of spikes and modes can be found in Ref. [28].

Since the phase information is lost in an intensity spectrum, one can-unfortunately-not simply retransform the spectral information into the time frame in order

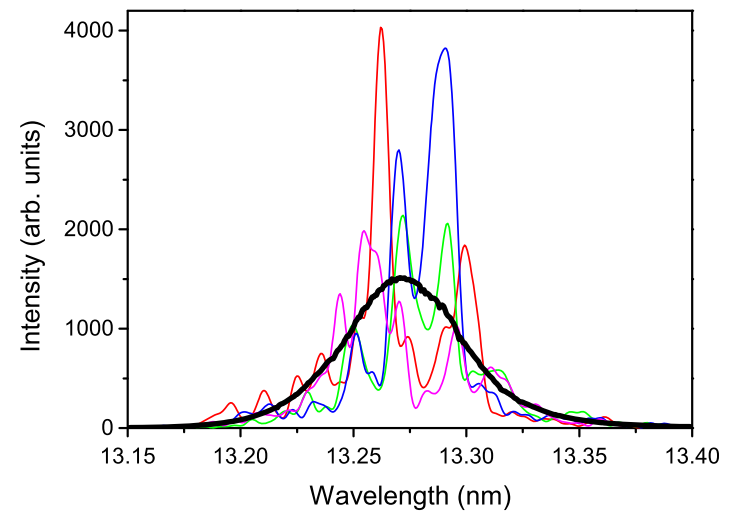

FIG. 8. Single-shot XUV spectra recorded for the $13.5 \mathrm{~nm}$ run (bunch 21) measured with the PG2 beam line. The spiky structure which is changing from pulse to pulse can easily be seen. The black line shows the average over hundreds of single-shot spectra.

to derive the photon pulse duration. However, a collection of single-shot spectra with sufficient resolution allows one to estimate the radiation pulse duration in two ways: first, by deriving the number of spikes (modes) from a single-shot spectrum measurement in combination with the coherence time (single peak width) estimated with Eq. (5). According to Eq. (6), the minimum photon pulse duration then is basically the number of modes multiplied by the coherence time. SASE FELs typically operate in the saturated regime where the FEL process is highly nonlinear and where a lengthening of the photon pulse duration occurs due to the already mentioned lasing in the tails of the electron bunch and due to slippage effects. Although this method of "mode counting" allows one to estimate the pulse duration on a shot-to-shot basis in a relatively easy manner-which is also very helpful for tuning the SASE FEL-there is no detailed investigation so far that this method still works reliably in saturation.

The second and more elaborate technique for average pulse duration determination exploits the analysis of the statistical properties of the SASE FEL spectra. Here, a spectral correlation analysis of a larger set of FEL spectra using the second-order spectral correlation function is carried out. A detailed description of the method and experimental results can be found in Ref. [54] for measurements at LCLS as well as in Refs. [55] (SACLA) and [56] (FLASH). In contrast to the first method, the spectral intensity correlation analysis has the tremendous advantage that the method is still applicable in the saturation regime which was confirmed by experiments and simulations (see Ref. [54]). As a consequence, the pulse duration can be determined any time the spectra are taken, and no additional FEL tuning (e.g., change to linear regime) is needed in contrast to the statistical approach described in Sec. IV A. Furthermore, even simple models [57] simulating the longitudinal shapes of the SASE FEL pulses, mainly using the measured spectra as experimental input, 
yield surprisingly realistic pulse shapes and provide valuable tools for the interpretation of experiments [15].

In summary, the advantage of the determination of the pulse duration by the spectral characteristics is that already a single spectral measurement allows under certain conditions an estimate of the photon pulse length. In addition, these single-shot XUV spectra are routinely obtained at FLASH by using the plane grating monochromator beam line PG2 $[58,59]$ in the spectrometer mode, with a resolving power of $\lambda / \Delta \lambda>10000$. On the other hand, this measurement cannot be carried out online during user operation, since operating the PGM beam line as a spectrometer hinders the photons to be sent downstream to a user experiment.

\section{Afterburner: Mapping SASE pulses in the optical range}

A completely different approach to obtain information on the photon pulse duration is the mapping of the XUV pulses into the optical wavelength range [60]. The SASE process modulates the electron beam and energy spread on the scale of the coherence length (in the submicron to few micrometer range). This leads to a characteristic energy modulation of the electron pulse in the longitudinal section, where lasing took part. The temporal width of this section is the XUV photon pulse duration. The modulated section can be mapped out by using a magnetic chicane and a radiator-in the FLASH case a terahertz undulator [61]. The energy modulation is transformed into a density modulation by the magnetic chicane. The subsequent terahertz undulator is tuned appropriately such that the density-modulated section of the bunch radiates coherently in the optical or near infrared (e.g., $800 \mathrm{~nm}$ ). Thus, the measurement of the XUV pulse is mapped into the optical or near infrared spectral range where a standard autocorrelator setup can be used. The method was demonstrated in the range $>30 \mathrm{fs}$ (FWHM). Investigations are ongoing to explore if the method can still be utilized for even shorter pulses. A detailed description of the method can be found in Ref. [60].

\section{XUV PULSE DURATION MEASUREMENTS: DIRECT METHODS}

\section{A. XUV autocorrelation in gas phase}

Intensity autocorrelation is one of the most straightforward methods to measure ultrashort pulse durations in the optical range. It is based on nonlinear processes occurring in crystals or two-photon diodes. Unfortunately, in the XUV spectral range no nonlinear processes in crystals are known at this time, and the band gap of two-photon diodes is insufficient. For these reasons, two-photon processes in rare gases are used as a nonlinear medium to realize intensity autocorrelations in the XUV regime [14,15]. The absorption of $n$ photons might lead to multiple ionization with the ion yields $Y^{(q+)}$ in a certain charge state $q$ being proportional to the intensity $I$ to the $n$th power $Y^{(q+)} \propto I^{n}$ for nonlinear reaction pathways [15]. Thus, measuring the respective ion yield for two pulses as a function of the relative delay represents an $n$ th-order autocorrelation. Consequently, the temporal width of the pulse can be extracted from an autocorrelation trace when the order of the process is known. Additionally, an assumption of a simple pulse structure and a known overall shape (Gaussian, Lorentzian, square, etc.) has to be made in order to reconstruct the pulse duration [14]. Furthermore, the partial coherence of the SASE FEL pulses can also manifest itself in the autocorrelation spectra, giving rise to a second, narrow autocorrelation peak on top of the structure related to the overall pulse length [62]. This narrow peak corresponds to the coherence time. Its appearance demonstrates that the temporal resolution of the autocorrelation technique can be as good as $1 \mathrm{fs}$.

For the whole spectral range of FLASH, nonlinear photo effects in rare gases are suitable. Neon, for example, might be ionized at $13.5 \mathrm{~nm}$ in a four-, six-, and eight-photon process to $\mathrm{Ne}^{4+}, \mathrm{Ne}^{5+}$, and $\mathrm{Ne}^{6+}$, respectively, including a direct two-photon ionization transition from $\mathrm{Ne}^{3+}$ to $\mathrm{Ne}^{4+}$, $\mathrm{Ne}^{4+}$ to $\mathrm{Ne}^{5+}$, and $\mathrm{Ne}^{5+}$ to $\mathrm{Ne}^{6+}$. However, these processes can be screened by alternative sequential one-photon ionization routes assisted by electron shakeup [63]. With additional transitions, nonlinear processes down to $7.9 \mathrm{~nm}$ are available with neon. To reach these high ionization states, high XUV intensities are necessary. However, at short wavelength and higher ionization states, some additional issues like space charge and saturation effects could occur. Therefore, the analysis and exact determination of the pulse duration must be extracted and simulated for every wavelength and intensity separately. Alternatively, the direct photoionization of helium is well defined in the range of 12.5-24 eV. However, already for the two-photon double ionization (40-54 eV), direct and sequential ionization paths compete and the evaluation of the measured pulse duration requires modeling of the processes [14].

To realize autocorrelation experiments in the XUV spectral range, the pulse is split into two identical replica. Since the XUV absorption cross section in every material is high, the radiation is absorbed within a few hundred nanometers. For this reason, the division is realized by geometrical wavefront splitting. Two setups have been pursued at FLASH: one at the beam line PG2 [64] and one at beam line BL2 [65]. Both are working with the same principle. A mirror with a sharp edge is moved under grazing incidence into the beam and reflects one-half of the beam to a defined beam path, while the second half misses the mirror surface and is reflected by a different set of mirrors. At the end of the setup, the beams are recombined and sent in the original direction. The mirrors of the different beam paths can be moved in a way to create a defined delay between both arms. Because of the grazing 


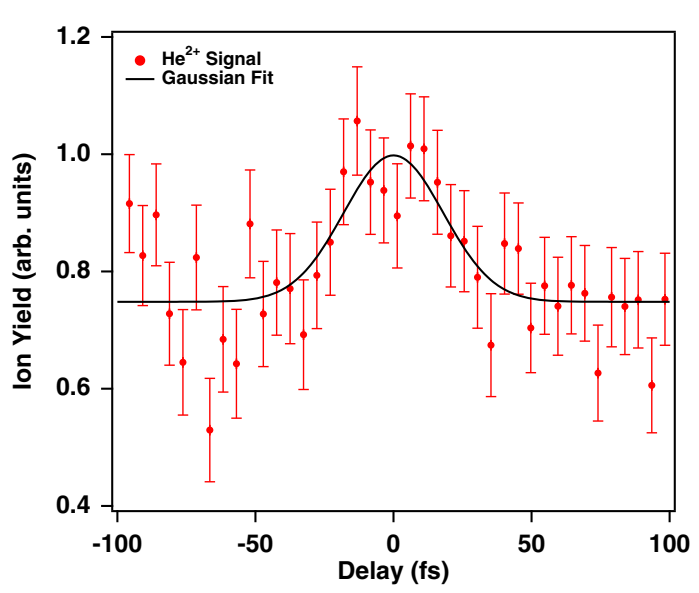

FIG. 9. Autocorrelation signal for helium averaged over all 30 bunches. The figure shows the $\mathrm{He}^{2+}$ signal resulting from a twophoton absorption. The fit yields a XUV pulse duration of $30 \pm$ 6 fs (FWHM). The data were recorded for an XUV wavelength of $24 \mathrm{~nm}$. For each data point, $\sim 80.000 \mathrm{XUV}$ pulses were summed.

incident angle and the used mirror coatings, a large spectral range can be transmitted and a relatively large delay can be scanned (in the range of $10 \mathrm{ps}$ ). To record the autocorrelation signal, the delay between the two replicas has to be scanned and the ion signal of the ionized gas is detected by a time-of-flight spectrometer [7]. The whole setup contains a high degree of complexity and extreme requirements on stability. The generation of the spatial overlap of the partial beams in the ionization volume is an experimental challenge of this method. Although it is a direct measurement of the XUV pulse duration for known ionization states, it requires averaging over many XUV pulses. The XUV pulse duration of the FEL may vary from shot to shot or within a bunch train, which cannot be resolved by a scanning setup. A single-pulse measurement would be possible with a single-shot autocorrelation technique as they are common in the optical range. For XUV pulses, single-shot concepts are currently under investigation. Figure 9 shows a typical autocorrelation trace recorded at $24 \mathrm{~nm}$ for the $\mathrm{He}^{2+}$ ions which shows a pulse duration of $30 \pm 6$ fs.

\section{B. Autocorrelation in semiconductors}

When semiconductors like silicon or gallium arsenide are excited by using XUV photons, one creates hot electrons which are known to change the reflectivity in the optical regime. This effect is extensively used to determine the exact temporal overlap of the XUV and optical pulses in pump-probe experiments [66-68]. Hence, one can expect that the excitation of hot electrons also changes the reflectivity of XUV photons.

For this technique, we have used a standard silicon sample covered with a silicon nitride $\left(\mathrm{Si}_{3} \mathrm{~N}_{4}\right)$ film. A radiation hard and fast silicon diode was used to measure the reflected XUV light. A bias voltage was applied in order to ensure a linear response of the diode over a large dynamic range. The sample was placed at an angle of $45^{\circ}$ with respect to the incoming and reflected photons. The experiment was carried out during the $13.5 \mathrm{~nm}$ run at the PG2 beam line using the permanently installed splitand-delay line [64]. The delay of the split-and-delay line was scanned at a slow speed of below $1 \mathrm{fs} / \mathrm{s}$. The temporal overlap was established beforehand by observing interference fringes $[26,27,64]$ on a beam monitor upstream. The recorded signal is shown in Fig. 10, which clearly shows a delay dependence which is as expected symmetric around $t_{0}$.

The interpretation of these data is, unfortunately, not straightforward. As was shown by using optical light as a probe, the excitation relevant for this probe decays on a picosecond time scale [66]. However, the measured data suggest that the relevant excitation for a XUV probe decays on a much faster time scale via Auger decay. In order to gain a qualitative understanding of the processes in the sample, we first focus on the delay values $t=0$ and $t \geq \tau_{\mathrm{XUV}}$. In the first case, the pulse can be treated as a single pulse which creates a signal $S_{0}$. For a delay time much greater than the pulse length and the decay time of the excitation, one pulse creates a signal $S_{\geq}$. Assuming that the photon pulse entering the split-and-delay unit is split into two equal parts, $S_{\geq}=S_{0} / 2$. Since we have two pulses and the diode is a slow detector, the measured diode signal is equal to the situation at $t=0$. In order to determine the

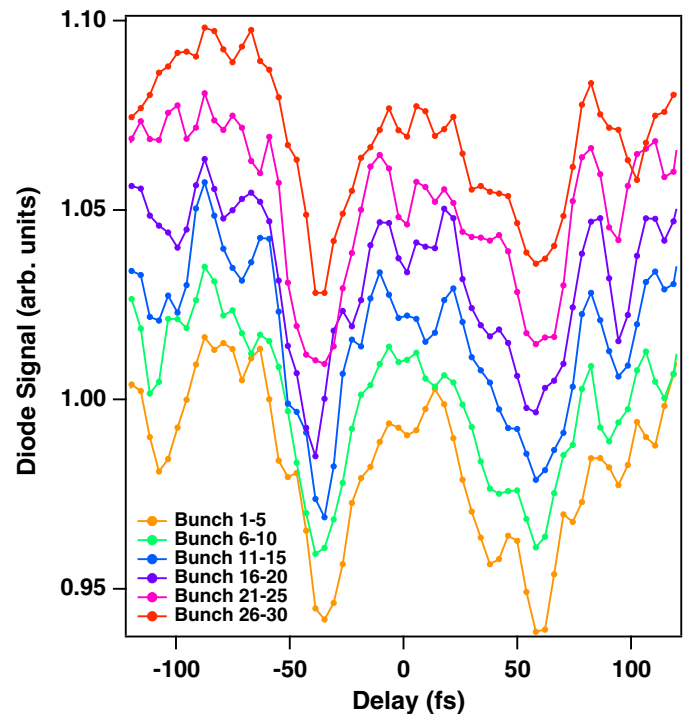

FIG. 10. Plotted is the XUV reflectivity of a solid target $\left(\mathrm{Si}_{3} \mathrm{~N}_{4}\right)$ as a function of the relative delay between the two pulses for groups of five bunches in the bunch train. Each data point is the average of about 210 single events giving an overall measurement time of about $5 \mathrm{~min}$ for the whole data set. The bunch-resolved (in groups of five bunches) autocorrelation signal for a solid target $\left(\mathrm{Si}_{3} \mathrm{~N}_{4}\right)$ is shown. For each data point, $210 \mathrm{XUV}$ pulses were summed. The figure shows the XUV reflectivity measured as a function of the delay between the two pulses. The data were recorded for a wavelength of $13.5 \mathrm{~nm}$. 
exact pulse length, one has to know the decay time of the excitation. A good approximation is a typical Auger decay time of 10 fs $($ Si $2 p)$.

In general, the change of XUV reflectivity is an effect of the hot electrons created by secondary processes after the ultrashort pulse hit the sample. The temporal resolution of this method is thus limited by the lifetimes of the involved processes and the scattering rates of the electrons in the semiconductor. These time scales are typically on the order of a few tens of femtoseconds.

\section{Cross-correlation with terahertz}

The direct techniques described so far are able only to determine averaged pulse shapes-which blur the spiky SASE nature completely. To map the temporal shape directly for each XUV pulse-without folding with itself or other pulses - into a measurable quantity, the so-called streaking technique can be used, where the ionizing XUV pulse is superimposed with an infrared light field with a longer periodicity as compared to the ionizing pulse length. The electrons created by the XUV pulse are immediately accelerated by the electromagnetic light field, whereby the resulting momentum change depends on the phase of the infrared field at the time of ionization [see Fig. 11 (upper panel)]. Thus the temporal profile of the ionizing XUV pulse is mapped onto photoelectron energies. The technique was first used in attosecond metrology where near infrared streaking fields were employed to determine the pulse duration of isolated attosecond pulses [69-72]. By choosing streaking fields with longer oscillation periods in the terahertz range, it was adapted for the analysis of femtosecond pulse durations $[16,17,73,74]$.

To measure the FLASH XUV photon pulses, a streaking wavelength of $\sim 100 \mu \mathrm{m}$ is required which can be delivered by the FLASH terahertz undulator [61]. The undulator is located downstream of the XUV undulators, and, thus, the terahertz and XUV pulses are generated by the same electron bunches. This leads to a stable phase relation between the terahertz and XUV pulses with a jitter of only a few femtoseconds [16].

In the experiment, photoelectron spectra are measured parallel, antiparallel, and perpendicular to the terahertz polarization. Along the terahertz polarization, the resulting photoelectron spectrum is "streaked," and thus the temporal shape of each XUV pulse is mapped into the energy domain. In the direction perpendicular to the terahertz polarization, the streaking influence is much less, and thus the (almost) undisturbed photoelectron spectrum can be obtained (see Fig. 11). This unstreaked spectrum resembles the SASE FEL wavelength distribution which is used for "normalizing" the streaked spectra. By comparing the streaking parallel and antiparallel to the terahertz polarization, the linear chirp of the FEL pulses can be determined. By using this method, a resolution of $\sim 10 \mathrm{fs}$ was
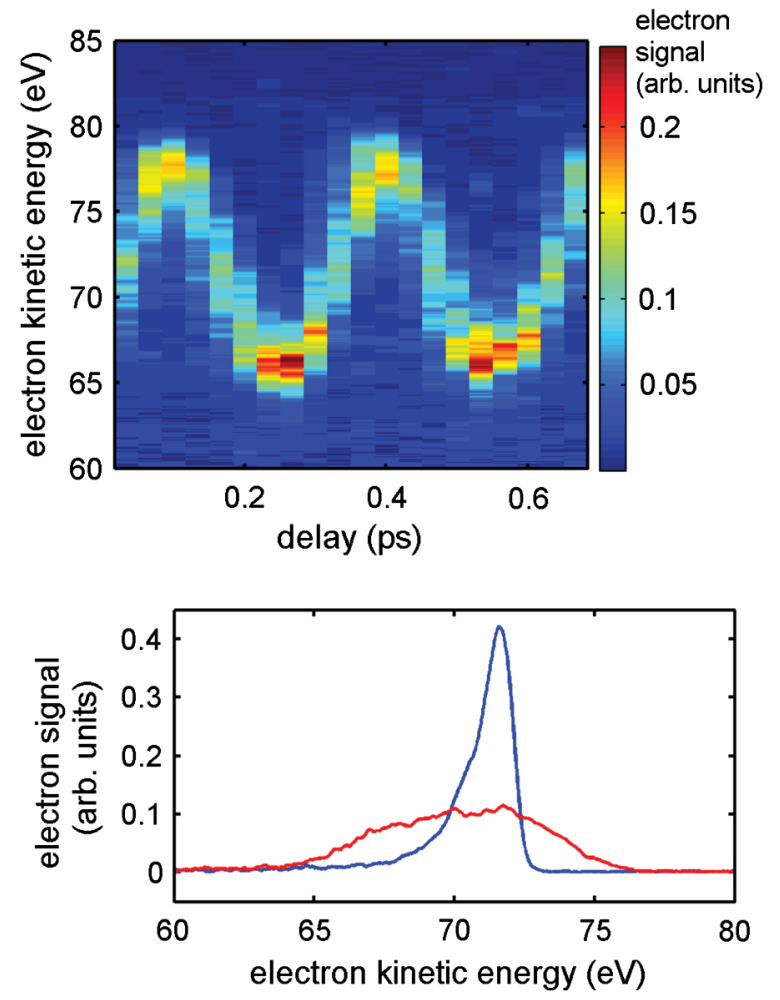

FIG. 11. Series of photoelectron spectra measured for different terahertz-XUV delays (upper panel). The energy shift of the electrons versus the X-ray/terahertz delay directly represents the vector potential of the terahertz field. Each spectrum was averaged over 20 shots. The lower panel shows the nonstreaked (blue line) and streaked (red line) photoelectron spectrum measured parallel to the terahertz polarization. The delay was set to a zero crossing of the terahertz vector potential $(\sim 0.35 \mathrm{ps}$ in the upper panel). Each spectrum was averaged over 500 shots.

demonstrated. For stronger streaking fields, a better resolution may be possible. For details, see Ref. [16].

Even though generated by the same electron bunch, the XUV and terahertz beam lines have a different path to the experimental setup, which needs to be compensated in order to achieve time overlap. In the present setup, the XUV pulses are delayed by a backfocusing mirror in respect to the terahertz pulses. The specific coating of this mirror currently limits the usable wavelength range to $\lambda \gtrsim 7 \mathrm{~nm}$. This unfortunate limitation can be overcome by using several remotely switchable mirrors or by using two electron bunches with a suitable distance of a few nanoseconds [75] to generate the XUV and terahertz pulses separated by a proper delay to compensate the arrival time difference. The resolution of the experiment depends on the spectral width of the XUV pulse and on the streaking speed and, thus, on the intensity and the wavelength of the terahertz pulse. Another difficulty of this method is that for very short photon pulses small electron bunch charges have to be used. For an electron bunch charge of less than $100 \mathrm{pC}$, the intensity of the 
terahertz pulses is so low that an accurate measurement is difficult.

An alternative approach, circumventing the wavelength and charge limitations, is the generation of the terahertz field using an optical (or near IR) laser to generate the terahertz streaking field as described in Ref. [17].

\section{COMPARISON OF THE METHODS}

Looking at the various techniques, it is obvious that there is a broad range from single-shot methods and those which require averaging over several, even up to tens of thousands of pulses. Finally, for SASE FELs the capability to measure each generated photon pulse on a single-shot basis is desirable due to the inherent shot-to-shot fluctuations of the pulse shape and its substructure. On the other hand, for technical reasons, averaging and indirect methods are often much simpler to realize and yield valuable information as well. Furthermore, given the burst mode structure of FLASH, one also has to distinguish between averaging experiments that can resolve the pulse duration of each pulse of the burst or have to average over all pulses in the train. Several techniques which are now used in an averaging mode are, in principle, able to deliver more detailed information on individual pulses with improved detectors in the future. Table I summarizes the present and potential options of the used techniques. In practice, averaging (scanning) techniques have also the disadvantage that they require significantly more beam time to provide a result. Their usefulness as tools to tune the accelerator is therefore limited. Fast, single-shot techniques are clearly preferable, especially if they could even be integrated into the FLASH feedback systems.

Because of the complexity of most methods, a simultaneous measurement of all techniques during the campaign was not possible. Therefore, care has been taken to keep the XUV pulse parameters as stable as possible during the measurements (5-6 shifts per wavelength). The electron beam was kept stable with various feedback systems. The most important ones in this context are the stabilization of bunch compression using the BCMs after the first and second bunch compressors. The BCMs resolve every single bunch in a train and are used to feedback the compression of the electron bunches. Other important feedbacks run on the beam energy and bunch charge. Because of the nature of the exponential amplification of the SASE process, we cannot precisely predict the influence of measurable electron bunch parameters to the photon pulse duration. Thus it is difficult to clearly distinguish between unresolved changes in the electron beam compression and remaining systematic errors. Therefore, the error bars shown in Figs. 12, 15, and 16 are only errors which can be attributed to measurement statistics.

Another complication is due to a possible systematic change in XUV-pulse duration along the bunch train. Some techniques can resolve individual pulses, some average over a bunch train or many trains, and others can use only one specific bunch of the train. Thus, for all pulse-resolving techniques, the measured results were classified into groups: A group of first bunches (numbers 1-10) and last bunches (numbers 20-30). The results of the measurements sorted according to first and last bunches are shown in Fig. 12 for the $13.5 \mathrm{~nm}$ run and in Fig. 16 for $24.0 \mathrm{~nm}$. Note that the analysis of the individual methods was done independently of other techniques and the gained pulse duration data were collected before the results of the other methods were presented to the rest of the team. The location of the different measurement techniques along the FEL is shown in Fig. 1.

TABLE I. Summary of acquisition modes of the pulse duration measurement techniques used in the experimental campaign. ss: single shot, every pulse generated is measured; pr: averaging technique, able to deliver the (average) pulse duration on every pulse in the burst (pulse resolving); av: averaging over the burst and over many successive shots. Parasitic is defined in a way that the measurement is not disturbing the beam and can be done in parallel to a user experiment.

\begin{tabular}{|c|c|c|c|}
\hline Method & Present acquisition mode & Possible upgrades & Parasitic \\
\hline TDS & $\mathrm{ss}^{1}$ & ss & $\mathrm{No}^{2}$ \\
\hline $\mathrm{BCM}$ & ss & ss & Yes \\
\hline Terahertz spectrometer CRISP & $\mathrm{pr}$ & $\mathrm{pr}$ & $\mathrm{No}^{2}$ \\
\hline XUV spectra & pr & pr & $\mathrm{No}^{3}$ \\
\hline XUV statistics & $\mathrm{pr}$ & $\mathrm{pr}$ & No \\
\hline Terahertz afterburner & av & ss & Yes \\
\hline XUV autocorrelation (gas phase) & av & $\operatorname{pr}\left(\mathrm{ss}^{4}\right)$ & No \\
\hline XUV autocorrelation (solid state) & $\mathrm{pr}$ & ss & No \\
\hline Terahertz streaking & ss & ss & $\mathrm{No}^{5}$ \\
\hline
\end{tabular}

\footnotetext{
${ }^{1}$ Arbitrary bunch out of bunch train can be measured.

${ }^{2}$ The bunch to be measured is destroyed, while other pulses from the bunch train can be used for experiments. If such a setup can be installed behind the XUV undulators, the measurement would be parasitic.

${ }^{3}$ A setup for parasitic XUV spectral measurements exists [10], however presently not with sufficient resolution.

${ }^{4}$ Ideas for a single-shot autocorrelator are currently under investigation.

${ }^{5}$ By combining (physically) the streaking setup with a user experiment, the streaking can be run in parasitic mode.
} 

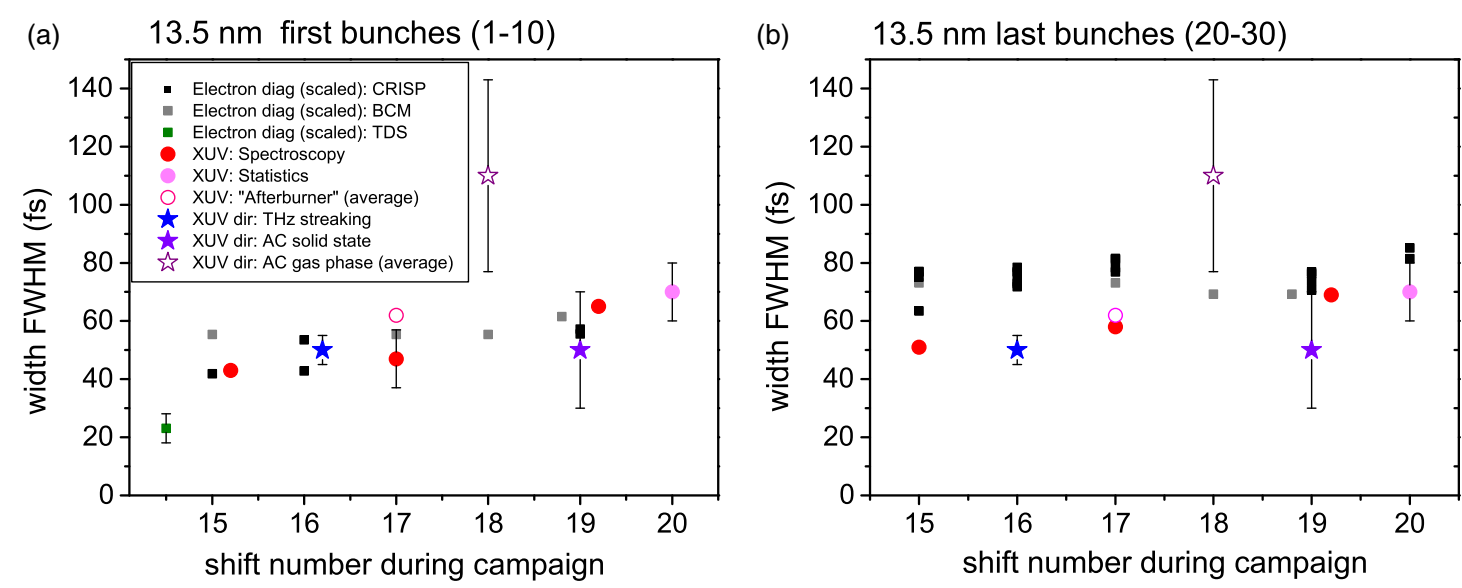

FIG. 12. XUV and electron bunch pulse duration (FWHM) measured during the $13.5 \mathrm{~nm}$ run. (a) shows the pulse durations for the first bunches (1-10) in the bunch train; (b) shows the pulse durations for the last bunches (20-30). The electron bunch duration is scaled for best fit with the average XUV pulse by a factor of 0.4 . The electron pulse measurements are indicated by a rectangular symbol, indirect photon pulse duration measurements by circles, and direct XUV measurements by stars. Furthermore, the pulse-resolved methods are plotted with solid symbols, while the averaging techniques are shown as open symbols. The horizontal axis indicates the shift number during the campaign. The first 15 shifts were used for setting up the FEL and the various experiments. One shift corresponds to $8 \mathrm{~h}$.

\section{A. Results of the $13.5 \mathrm{~nm}$ run}

The aim was to adjust the electron beam parameters such that the XUV pulse duration is about 50 fs (FWHM), as predicted from FEL simulations. For this, the single-bunch charge was adjusted to $150 \mathrm{pC}$. The usual tuning of beam optics, orbit in the undulator, and compression settings in the two bunch compressors was applied to achieve SASE radiation close to and at saturation. The tuning parameters were kept constant over the run. The pulse energy was about $50 \mu \mathrm{J}$ (for each single pulse). The 30 bunches per train with a temporal spacing of $4 \mu \mathrm{s}(250 \mathrm{kHz})$ have been chosen in order to allow the different techniques to use averaging over the train or to show their capabilities to resolve individual bunches. Within the statistical fluctuations of SASE, the pulse energy was the same for all pulses in the bunch train.

Figure 12 shows the results for the XUV and the electron pulse duration measurements of the $13.5 \mathrm{~nm}$ run. The pulse durations determined by the different techniques are in rather good agreement, with two exceptions (TDS and XUV autocorrelation).

The comparison in detail: The photon-based methods (circles and stars in Fig. 12) show a remarkable agreement for direct and indirect methods (except the gas phase autocorrelation). Within the same shift or neighboring shifts, there is only a difference of a few femtoseconds ( $\pm 10 \mathrm{fs})$ between methods. This is true for the first [Fig. 12(a)] as well as for the last bunches [Fig. 12(b)]. Thus the pulse duration was constant over the bunch train within $50 \pm 10 \mathrm{fs}$ as anticipated from the settings of the accelerator. There is, however, a consistent slight elongation of the pulse duration from shift to shift from $50 \mathrm{fs}$ average pulse duration to $\sim 60 \mathrm{fs}$ after 5 shifts $(40 \mathrm{~h})$.
Concerning the observed deviations, the autocorrelation measurement (in gas phase) shows a considerably longer pulse duration with large error bars. This can be attributed to experimental problems resulting in a very low count rate in the measurement. Here, the counting statistics do not allow a reliable conclusion of the pulse duration. This is not a principle limitation for the method but rather due to experimental problems during this campaign.

The much shorter bunches measured by the TDS can be explained by the slightly different conditions for the TDS measurements. For technical reasons, the TDS could be used only with two bunches, which is different from the conditions for the other methods where 30 bunches were used in a bunch train.

The given error bars are derived from successive measurements for the same settings. Thus the shown errors are due to statistical fluctuations. Techniques with good signal to noise show therefore rather small (statistical) error bars. It is certainly still possible that systematic errors are present which are not included in Figs. 12, 15, and 16 since they are simply not known. Part of the study was to identify such systematic errors. But looking at the $13.5 \mathrm{~nm}$ data, there are no indications of systematic large deviations.

The comparison between photon-based and electronbased diagnostics also shows a quite good agreement concerning slow drifts and the overall constant conditions during the run. As discussed in Sec. II, the XUV pulse is typically shorter than the electron pulse.

According to Fig. 2, the measured electron bunch durations have to be scaled by a factor of about 0.6 around saturation. However, in order to fit the measured electron pulse duration to the XUV pulse length measurements, a factor of 0.4 led to the best overlap. Thus, an even smaller 
part of the electron pulse was lasing as compared to the estimation made in Sec. II.

Looking at the later (20-30) bunches within the bunch train [Fig. 12(b)], the electron pulse diagnostics (CRISP and $\mathrm{BCM}$ ) show a longer pulse duration of the electrons of $60-80 \mathrm{fs}$ (scaled by 0.4 ) instead of $40-60 \mathrm{fs}$ (scaled by 0.4 ) for the first bunches [Fig. 12(a)], while the photon-based measurements show the same pulse duration for the first and the last pulses. Thus a scaling factor of $\sim 0.3$ would fit better leading to a still smaller lasing fraction of the electron bunch.

Already from this observation, it becomes clear that the determination of a scaling factor between electron beam diagnostic and XUV pulse length measurements needs more information of the electron bunch than only the pulse duration.

\section{B. Start-to-end simulation for $13.5 \mathrm{~nm}$}

SASE performance at FELs is strongly dependent on the electron beam quality. In the one-dimensional theory, it enters into the FEL parameter $\rho$ through the beam current density $j_{0}$ [compare Eq. (1)]. In reality, additional effects such as beam energy spread, beam focusing and emittance, space charge forces, and radiation diffraction play a role as well. The emitted SASE pulse depends significantly on the details of the six-dimensional phase space distribution of the electron bunch.

While the FLASH injector is optimized for very good beam quality, collective effects during acceleration, compression, and beam transport may decrease the beam quality and thus influence SASE performance. Beam dynamics, especially at strong compression, is very complicated. Therefore, in order to include these effects, dedicated start-to-end simulations have been performed for the machine settings used during this campaign.

For this, a combination of specialized simulation codes has been used to model the FEL FLASH from the gun to the end of the undulator section. The code ASTRA [76] was used for the main linac to include longitudinal as well as transverse space charge forces on the bunch, which cannot be neglected for the beam energy range used at FLASH. For the bunch compressors and dogleg, the code CSRTRACK [77] has been used which takes into account coherent synchrotron radiation effects.

The simulated bunch at the end of the linac was compared to the longitudinal current profile measured with the terahertz spectrometer CRISP, which is described in Sec. III B 1. Because of an uncertainty of the reference phase of the rf, the actual phase of the accelerating modules is known only to some degree. As a consequence, the rf phase in the simulation has been scanned to find the best match between the longitudinal current distribution resulting from CRISP measurement and simulation. At FLASH the phase with the strongest influence on the longitudinal current distribution of the bunch is the phase of the first accelerating module downstream of the gun (ACC1). For complexity reasons, the phase scanning for the simulations was restricted to the $\mathrm{ACC} 1$ phase while the influence of a drift in the other phases on the current profile of the bunch was neglected. This may result in a small deviation between the simulated and measured electron bunches.

Finally, the 3D time-dependent simulation code GENESIS 1.3 [78] was used to model the FEL process of the simulated electron bunch in the undulator section. Because of the relatively low peak current at the end of the FLASH linac, the influence of longitudinal space charge forces on the FEL performance is expected to be negligible, and thus they were not included in this simulation.

A perfect matching of the electron bunch to the undulator has not been assumed for these studies. Instead, the optics used for this simulation is - to our best knowledge-the exact optics that was in the machine during the measurements presented in this publication. It is therefore important to discuss the matching of the beam.

For optimum lasing process the bunch-or, to be more precise, the beta function, describing the external focusing applied on the bunch - has to be matched to the design parameters. This can be described by the beta-matching parameter BMAG $[79,80]$, with

$$
\mathrm{BMAG}=0.5\left(\beta \gamma_{0}-2 \alpha \alpha_{0}+\beta_{0} \gamma\right)
$$

where $\beta$ is the beta function, $\alpha=-\frac{\beta^{\prime}}{2}, \beta^{\prime}=d \beta / d z$, and $\gamma=\left(1+\alpha^{2}\right) / \beta$. The index 0 denotes the design values. The beam is matched to the machine if $\mathrm{BMAG}=1$, while BMAG $>1$ denotes a beam that is not well matched.

The matching may vary for different parts of the electron bunch. It is therefore important to consider it for different longitudinal slices along the bunch. The slice matching parameter of the simulated electron bunch corresponding to the $13.5 \mathrm{~nm}$ run is given in Fig. 13 (top). It can be seen that the bunch is well matched only around a longitudinal position $z=0.02 \mathrm{~mm}$, situated at the rising slope of the current profile. The corresponding slice emittance and spot size at the beginning of the undulator section are shown in Fig. 14. The slice energy spread is almost constant over the bunch. The resulting simulated SASE pulse is given in Fig. 13 (bottom). Only the well-matched part of the bunch contributes significantly to the lasing process. The SASE pulse emitted by this electron bunch is therefore smaller than expected by the simple assumption of a factor 0.6 between electron bunch and photon pulse duration (compare Fig. 2). This observation is in good agreement with the experimental findings for the $13.5 \mathrm{~nm}$ run (compare Fig. 12).

Knowledge of the longitudinal current profile alone is therefore not sufficient for a good estimation of the resulting SASE pulse duration. Additional information about slice properties of the bunch is required as well. 

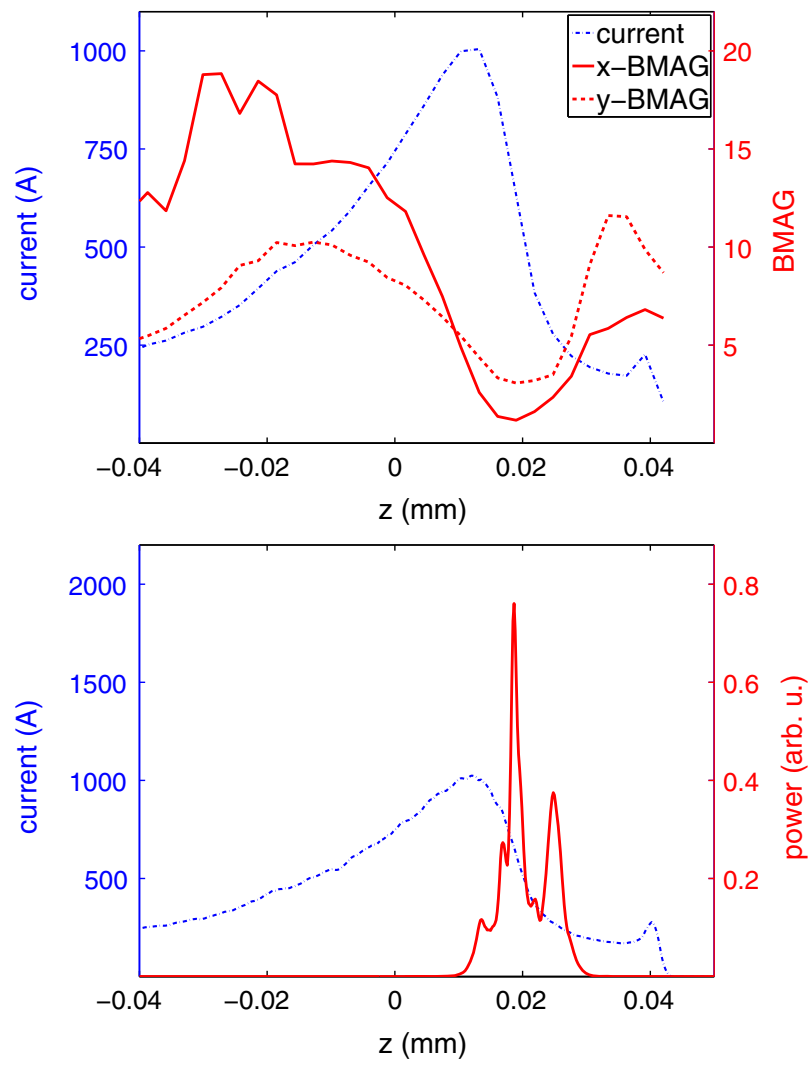

FIG. 13. Matching parameter BMAG as a function of the longitudinal position within the bunch (top) and simulated longitudinal SASE pulse distribution (bottom) for the start-toend simulation corresponding to the machine settings of the $13.5 \mathrm{~nm}$ run. The head of the bunch is to the right-hand side of the figure $(0.02 \mathrm{~mm}$ corresponds to $66 \mathrm{fs})$.

\section{Results of the $24.0 \mathrm{~nm}$ run}

As for the $13.5 \mathrm{~nm}$ run, the electron beam parameters have been adjusted to yield a XUV pulse duration of $\sim 50$ fs. The single-bunch charge was adjusted to $130 \mathrm{pC}$. Again, 30 bunches in the train were used with a distance of $4 \mu \mathrm{s}(250 \mathrm{kHz})$. SASE radiation was tuned to saturation and kept constant over the run.

In contrast to the $13.5 \mathrm{~nm}$ case, where the compression was set up in a way that all bunches in the bunch train have about the same pulse duration (with small deviations as discussed above), for $24 \mathrm{~nm}$ the tuning was such that the pulse duration measured by the terahertz electron pulse length diagnostic CRISP showed a linear decrease of the pulse duration (see black squares in Fig. 15) from 80 fs (in this case scaled by 0.66 as discussed below) for the first bunch to 20-30 fs (scaled by 0.66) for the last bunches. Thus, the last electron bunches in the bunch train were a factor of 3-4 shorter than the first ones according to CRISP. This pattern stayed constant for the whole $24 \mathrm{~nm}$ run. The changes during the bunch train were also visible in the XUV pulse energy of $80 \mu \mathrm{J}$ for the first bunches with a drop to about $50 \mu \mathrm{J}$ for the last ones. This behavior is expected.
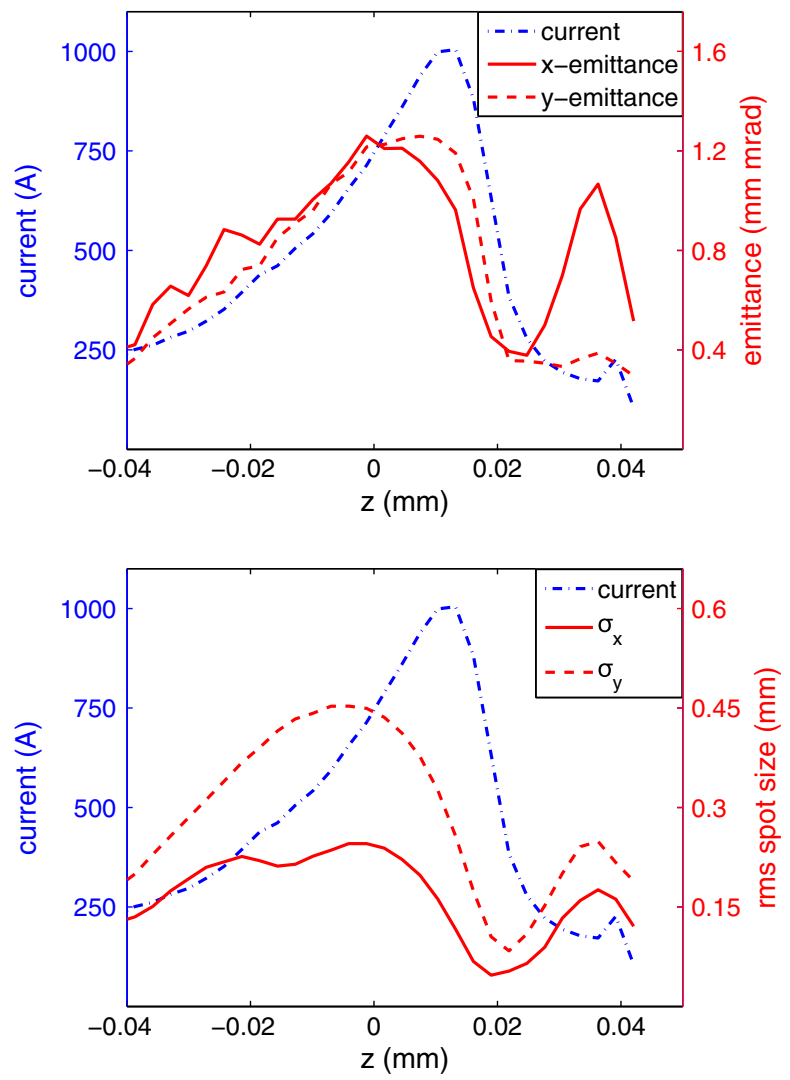

FIG. 14. Normalized slice emittance (top) and rms slice spot size at the beginning of the undulator section (bottom), resulting from start-to-end simulation for $13.5 \mathrm{~nm}$.

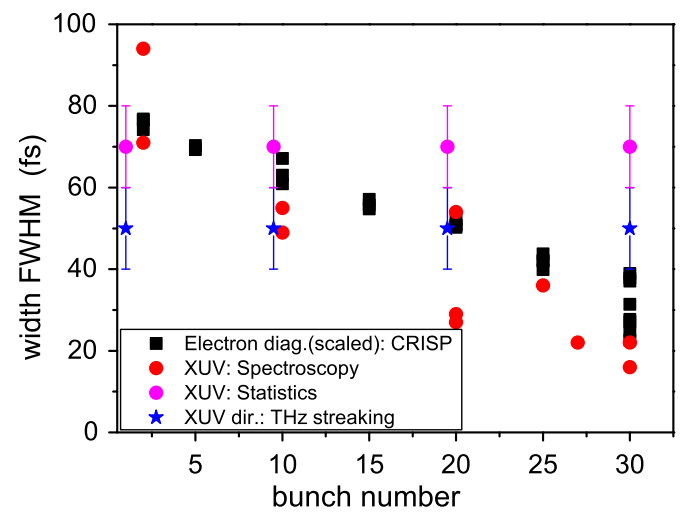

FIG. 15. XUV and electron bunch duration (FWHM) measurements of the pulse-resolved methods for the $24 \mathrm{~nm}$ run. All measurements of the run have been plotted as a function of the bunch number within the bunch train. The measurements result from different shifts (see Fig. 16). While CRISP (the electron duration is scaled by 0.66) and spectral analysis see a strong decrease of the pulse duration, terahertz streaking and statistical analysis measure no change of the pulse duration within the bunch train. 

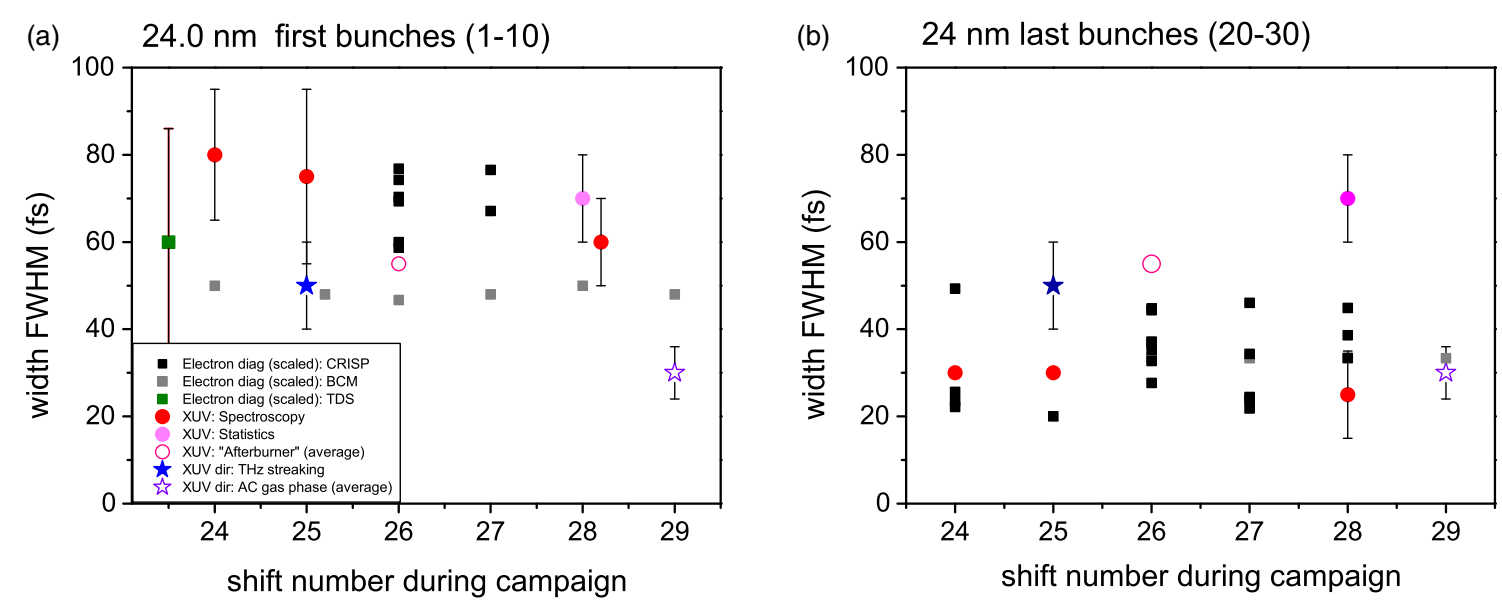

FIG. 16. XUV and electron bunch duration (FWHM) measurements during the $24 \mathrm{~nm}$ run. (a) shows the pulse durations for the first bunches (1-10) in the bunch train; (b) shows the pulse durations for the last bunches (20-30). The electron bunch duration is scaled by a factor of 0.66 to match the average electron pulse duration to the photon pulses. Electron pulse measurements are indicated by a rectangular symbol, indirect photon pulse duration measurements by circles, and direct XUV measurements by stars. Furthermore, the pulse-resolved methods are plotted with solid symbols, while the averaging techniques are shown as open symbols. The horizontal axis indicates the shift number during the campaign. A shift corresponds to $8 \mathrm{~h}$.

Shorter pulses yield less pulse energy than longer ones for the same level of saturation. Since every temporal spike contains on average the same energy, a longer pulse with more spikes or modes contains more pulse energy. A similar mode of operation was also used in Ref. [81].

The scaling factor between electron pulse length and photon pulse duration in the $24 \mathrm{~nm}$ case matches better with the expected value $\sim 0.6$. The scaling factor was chosen as 0.66 , which results in a minimum deviation between photon-based pulse durations and the scaled electron pulse durations.

The machine setting which resulted in the strongly varying pulse duration over the bunch train was chosen in order to have a broad range of pulse durations which can test the measurement range of the different (bunch-resolved) methods. Figure 16 shows the results for the measured pulse durations at $24 \mathrm{~nm}$, plotted in the same way as Fig. 12 for $13.5 \mathrm{~nm}$. In contrast to the $13.5 \mathrm{~nm}$ case, here the scatter between the different methods is much larger. This can be explained to some extent due to the varying pulse duration along the bunch train, since the pulse duration already changes within the 10-bunch window shown. Even more, several techniques average over all 30 bunches and deliver only a (sometimes weighted) average pulse duration. However, even the pulse-resolved techniques differ in the presented case. Plotting the pulse durations measured by the pulse-resolved techniques (CRISP, statistics, spectroscopy, and terahertz streaking) as a function of the pulse number reveals a distinct difference between the methods as shown in Fig. 15. While there is a good agreement between CRISP and the spectral analysis showing a decrease of the pulse duration of a factor 3-4 between the first and the last pulse, on the other hand, the statistical analysis and the terahertz streaking show no change within the error bars over the bunch train.

The reason for the discrepancies is not known, and to disentangle the various contributions afterwards is not entirely possible. In the case of strongly varying electron parameters within the bunch train, the pulse durations and shapes as well as the chirp may be considerably different for different parts of the bunch train. In this case, a much more detailed analysis of the different techniques has to be performed, and methods relying on the reconstruction based on certain bunch shapes (e.g., Gaussian) have to be taken with care. More detailed start-to-end simulations are currently being performed to gain more insight.

\section{SUMMARY}

One of the most challenging tasks for the FEL photon diagnostics is the precise determination of the FEL pulse duration. In the presented study, we used nine different techniques to determine the XUV pulse length either directly by measuring the XUV photon pulse at the end of the beam line or indirectly by the measurement of related parameters and the subsequent modeling of the initial XUV pulse duration.

For the measurements that were performed at $13.5 \mathrm{~nm}$, the FEL was tuned such that all pulses in the bunch train had roughly the same electron bunch and XUV pulse parameters. For this case, a remarkably good agreement between the methods was found. Most of all, it was shown that all used indirect methods reveal the same results as the direct methods, and thus the assumptions made for the analysis of the indirect methods seem to be valid for this 
case. On the other hand, when the electron pulse and XUV parameters are significantly changing within the bunch train, as in the $24 \mathrm{~nm}$ case, there is a strong deviation between different methods. While some methods show a significant change in pulse duration within the bunch train, other methods do not observe any change within their error bars. Here it is difficult to judge which method can be trusted to what extent. Most of all, this is problematic when several techniques in addition to the complicated pulse structures have experimental problems leading to low signal-to-noise ratios.

While in SASE mode of operation the photon pulse is typically shorter than the total length of the electron bunch from which it is generated, the assumption of a factor 0.6 between the two can be used only as a very simple rule of thumb for first estimations. As the start-to-end simulation showed, the factor can be substantially smaller depending on the accelerator settings. Because of the complicated beam dynamics in the energy range FLASH is working in, parameters like slice emittance and energy spread also have to be taken into account as well. Up to now, we can only state that a universal scaling factor between electron and photon pulses could not be determined for FLASH. It will be therefore important to not only measure the current profile of different bunches along the bunch train, but additional diagnostics is required to positively identify the lasing part of the bunch.

In conclusion, for FLASH we will further develop and automatize the electron-based diagnostics as a monitor for changes within a bunch train as well as for long term drifts in the pulse duration. In order to use the electron pulse duration techniques to state reliable XUV pulse durations, more investigations on the various dependencies of the scaling factor on the wavelength and other FEL parameters have to be undertaken. For the normal operation mode of FLASH (13.5 nm settings), the indirect methods showed their capability to measure the pulse durations with much less effort compared to the direct methods. Thus we will further automatize the usage of terahertz afterburner measurements and spectral analysis as future standard diagnostics. Finally, for SASE FELs like FLASH it is essential to also develop tools being able to deliver on a single-shot basis the pulse profile and the substructure as well as the arrival time in respect to an external laser source. The methods considered for future development are based on the XUVinduced reflectivity changes (this method was not part of the study; see, e.g., Refs. $[18,82])$ and terahertz streaking techniques (in particular, with optical laser-based terahertz sources as described in Ref. [17]).

A completely different approach, seeding FELs with external sources, will provide a much better defined pulse duration (and shape) and in the future will certainly aid the next generation of time-resolved and nonlinear physics with more precision as well [83-85].

\section{ACKNOWLEDGMENTS}

The authors acknowledge the work of the scientific and technical team at FLASH. We thank the European Union Seventh Framework Program (FP7/2007-2013) under Grant Agreement No. 283745 (CRISP) for funding as well as the BMBF funding via the priority program FSP 301 "FLASH."

[1] Y. H. Jiang, A. Rudenko, O. Herrwerth, L. Foucar, M. Kurka, K. U. Kuehnel, M. Lezius, M. F. Kling, J. van Tilborg, A. Belkacem, K. Ueda, S. Duesterer, R. Treusch, C. D. Schroeter, R. Moshammer, and J. Ullrich, Phys. Rev. Lett. 105, 263002 (2010).

[2] C. M. Günther, B. Pfau, R. Mitzner, B. Siemer, S. Roling, H. Zacharias, O. Kutz, I. Rudolph, D. Schondelmaier, R. Treusch, and S. Eisebitt, Nat. Photonics 5, 99 (2011).

[3] K. Schnorr et al., Phys. Rev. Lett. 111, 093402 (2013).

[4] L. Redecke et al., Science 339, 227 (2013).

[5] M. Richter, S. V. Bobashev, A. A. Sorokin, and K. Tiedtke, J. Phys. B 43, 194005 (2010).

[6] L. Young et al., Nature (London) 466, 56 (2010).

[7] A. A. Sorokin, S. V. Bobashev, T. Feigl, K. Tiedtke, H. Wabnitz, and M. Richter, Phys. Rev. Lett. 99, 213002 (2007).

[8] M. Richter et al., Appl. Phys. Lett. 83, 2970 (2003).

[9] K. Tiedtke et al., J. Appl. Phys. 103, 094511 (2008).

[10] G. Brenner et al., Nucl. Instrum. Methods Phys. Res., Sect. A 635, S99 (2011).

[11] A. A. Sorokin et al., Appl. Phys. Lett. 89, 221114 (2006).

[12] W. Ackermann et al., Nat. Photonics 1, 336 (2007).

[13] S. Schreiber, B. Faatz, J. Feldhaus, K. Honkavaara, R. Treusch, M. Vogt, and J. Rossbach, in Proceedings of the 32nd Free Electron Laser Conference, Malmö, Sweden (Max-lab, Sweden, 2010), TUOBI2.

[14] R. Mitzner, A. A. Sorokin, B. Siemer, S. Roling, M. Rutkowski, H. Zacharias, M. Neeb, T. Noll, F. Siewert, W. Eberhardt, M. Richter, P. Juranic, K. Tiedtke, and J. Feldhaus, Phys. Rev. A 80, 025402 (2009).

[15] Y. H. Jiang, T. Pfeifer, A. Rudenko, O. Herrwerth, L. Foucar, M. Kurka, K. U. Kühnel, M. Lezius, M. F. Kling, X. Liu, K. Ueda, S. Düsterer, R. Treusch, C. D. Schröter, R. Moshammer, and J. Ullrich, Phys. Rev. A 82, 041403(R) (2010).

[16] U. Frühling, M. Wieland, M. Gensch, T. Gebert, B. Schütte, M. Krikunova, R. Kalms, F. Budzyn, O. Grimm, J. Rossbach, E. Plönjes, and M. Drescher, Nat. Photonics 3, 523 (2009).

[17] I. Grguras, A. R. Maier, C. Behrens, T. Mazza, T. J. Kelly, P. Radcliffe, S. Duesterer, A. K. Kazansky, N. M. Kabachnik, Th. Tschentscher, J. T. Costello, M. Meyer, M. C. Hoffmann, H. Schlarb, and A. L. Cavalieri, Nat. Photonics 6, 852 (2012).

[18] R. Riedel, A. Al-Shemmary, M. Gensch, T. Golz, M. Harmand, N. Medvedev, M. J. Prandolini, K. SokolowskiTinten, S. Toleikis, U. Wegner, B. Ziaja, N. Stojanovic, and F. Tavella, Nat. Commun. 4, 1731 (2013). 
[19] C. Behrens, N. Gerasimova, Ch. Gerth, B. Schmidt, E. A. Schneidmiller, S. Serkez, S. Wesch, and M. V. Yurkov, Phys. Rev. ST Accel. Beams 15, 030707 (2012).

[20] R. Bonifacio, C. Pellegrini, and L. M. Narducci, Opt. Commun. 50, 373 (1984).

[21] R. Bonifacio, L. De Salvo, P. Pierini, N. Piovella, and C. Pellegrini, Phys. Rev. Lett. 73, 70 (1994).

[22] E. L. Saldin, E. A. Schneidmiller, and M. V. Yurkov, Opt. Commun. 148, 383 (1998).

[23] E. L. Saldin, E. A. Schneidmiller, and M. V. Yurkov, The Physics of Free Electron Lasers (Springer, Berlin, 2000).

[24] E. L. Saldin, E. A. Schneidmiller, and M. V. Yurkov, Nucl. Instrum. Methods Phys. Res., Sect. A 429, 233 (1999).

[25] R. Mitzner, B. Siemer, M. Neeb, T. Noll, F. Siewert, S. Roling, M. Rutkowski, A. A. Sorokin, M. Richter, P. Juranic, K. Tiedtke, J. Feldhaus, W. Eberhardt, and H. Zacharias, Opt. Express 16, 19909 (2008).

[26] W. F. Schlotter, F. Sorgenfrei, T. Beeck, M. Beye, S. Gieschen, H. Meyer, M. Nagasono, A. Föhlisch, and W. Wurth, Opt. Lett. 35, 372 (2010).

[27] A. Singer, F. Sorgenfrei, A. P. Mancuso, N. Gerasimova, O. M. Yefanov, J. Gulden, T. Gorniak, T. Senkbeil, A. Sakdinawat, Y. Liu, D. Atwood, S. Dziarzhytski, D. D. Mai, R. Treusch, E. Weckert, T. Salditt, A. Rosenhahn, W. Wurth, and I. A. Vartanyants, Opt. Express 20, 17480 (2012).

[28] S. Krinsky and R. L. Gluckstern, Phys. Rev. ST Accel. Beams 6, 050701 (2003).

[29] K. Tiedtke et al., New J. Phys. 11, 023029 (2009).

[30] S. Schreiber et al., in Proceedings of the 28th Free Electron Laser Conference, Berlin, Germany (BESSY, Berlin, 2006), THPPH017.

[31] S. Schreiber, S. Lederer, I. Hansen, H.-H. Sahling, P. Michelato, L. Monaco, and D. Sertore, in Proceedings of the 3rd International Particle Accelerator Conference, New Orleans, 2012 (IEEE, Piscataway, NJ, 2012), p. 625.

[32] I. Will, H. Templin, S. Schreiber, and W. Sandner, Opt. Express 19, 23770 (2011).

[33] F. Lohl et al., Phys. Rev. Lett. 104, 144801 (2010).

[34] X. J. Wang, in Proceedings of the 18th Particle Accelerator Conference, New York, 1999 (IEEE, New York, 1999), pp. 229-233.

[35] R. Akre, L. Bentson, P. Emma, and P. Krejcik, in Proceedings of the 19th Particle Accelerator Conference, Chicago, IL, 2001 (IEEE, Piscataway, NJ, 2001), pp. 2353-2355.

[36] R. H. Miller, R. F. Koontz, and D. D. Tsang, IEEE Trans. Nucl. Sci. 12, 804 (1965).

[37] O. H. Altenmueller, R. R. Larsen, and G. A. Loew, Rev. Sci. Instrum. 35, 438 (1964).

[38] C. Behrens, F.-J. Decker, Y. Ding, V. A. Dolgashev, J. Frisch, Z. Huang, P. Krejcik, H. Loos, A. Lutman, T. J. Maxwell, J. Turner, J. Wang, M.-H. Wang, J. Welch, and J. Wu, Nat. Commun. 5, 3762 (2014).

[39] M. Röhrs, Ch. Gerth, H. Schlarb, B. Schmidt, and P. Schmüser, Phys. Rev. ST Accel. Beams 12, 050704 (2009).

[40] C. Behrens and C. Gerth, in Proceedings of the 9th European Workshop on Beam Diagnostics and Instrumentation for Particle Accelerators, Basel, Switzerland, 2009 (PSI, Basel, 2009), pp. 269-271.
[41] S. Casalbuoni, B. Schmidt, P. Schmuser, V. Arsov, and S. Wesch, Phys. Rev. ST Accel. Beams 12, 030705 (2009).

[42] S. Wesch, B. Schmidt, C. Behrens, H. Delsim-Hashemi, and P. Schmüser, Nucl. Instrum. Methods Phys. Res., Sect. A 665, 40 (2011).

[43] R. Lai and A. Sievers, Nucl. Instrum. Methods Phys. Res., Sect. A 397, 221 (1997).

[44] E. Hass et al., in Proceeding of SPIE, Vol. 8778, (SPIE, Cardiff, UK, 2013).

[45] O. Grimm, in Proceedings of the 22nd Particle Accelerator Conference, PAC-2007, Albuquerque, NM (IEEE, New York, 2007), THYC02.

[46] H. Loos et al., in Proceedings of the 22nd Particle Accelerator Conference, PAC-2007, Albuquerque, NM (IEEE, New York, 2007), FRPMS071.

[47] C. Behrens et al., in Proceedings of the International Particle Accelerator Conference, Kyoto, Japan (ICR, Kyoto, 2010), MOPD090.

[48] S. Wesch, Ph.D. thesis, University of Hamburg, 2012, Report No. DESY-THESIS-12-052.

[49] V. Ayvazyan et al., Eur. Phys. J. D 20, 149 (2002).

[50] J. Wu et al., in Proceedings of the 32nd Free Electron Laser Conference, Malmö, Sweden (Max-lab, Sweden, 2010), MOPC14.

[51] A. Bytchkov et al., Nucl. Instrum. Methods Phys. Res., Sect. A 528, 254 (2004).

[52] L. Bittner et al., in Proceedings of the 29th Free Electron Laser Conference, Novosibirsk, Russia (BINP, Novosibirsk, 2007), WEPPH007.

[53] S. Roling, B. Siemer, M. Wöstmann, H. Zacharias, R. Mitzner, A. Singer, K. Tiedtke, and I. A. Vartanyants, Phys. Rev. ST Accel. Beams 14, 080701 (2011).

[54] A. A. Lutman, Y. Ding, Y. Feng, Z. Huang, M. Messerschmidt, J. Wu, and J. Krzywinski, Phys. Rev. ST Accel. Beams 15, 030705 (2012).

[55] Y. Inubushi, K. Tono, T. Togashi, T. Sato, T. Hatsui, T. Kameshima, K. Togawa, T. Hara, T. Tanaka, H. Tanaka, T. Ishikawa, and M. Yabashi, Phys. Rev. Lett. 109, 144801 (2012).

[56] N. Gerasimova et al. (to be published).

[57] T. Pfeifer, Y. Jiang, S. Düsterer, R. Moshammer, and J. Ullrich, Opt. Lett. 35, 3441 (2010).

[58] M. Martins, M. Wellhoefer, J. T. Hoeft, W. Wurth, J. Feldhaus, and R. Follath, Rev. Sci. Instrum. 77, 115108 (2006).

[59] N. Gerasimova, S. Dziarzhytski, and J. Feldhaus, J. Mod. Opt. 58, 1480 (2011).

[60] E. L. Saldin, E. A. Schneidmiller, and M. V. Yurkov, Phys. Rev. ST Accel. Beams 13, 030701 (2010).

[61] M. Gensch, L. Bittner, A. Chesnov, H. Delsim-Hashemi, M. Drescher, B. Faatz, J. Feldhaus, U. Fruehling, G. A. Geloni, Ch. Gerth, O. Grimm, U. Hahn, M. Hesse, S. Kapitzki, V. Kocharyan, O. Kozlov, E. Matyushevsky, N. Morozov, D. Petrov, E. Ploenjes, M. Roehling, J. Rossbach, E. L. Saldin, B. Schmidt, P. Schmueser, E. A. Schneidmiller, E. Syresin, A. Willner, and M. V. Yurkov, Infrared Phys. Technol. 51, 423 (2008).

[62] A. Senftleben, T. Pfeifer, K. Schnorr, K. Meyer, Y. H. Jiang, A. Rudenko, O. Herrwerth, L. Foucar, M. Kurka, K. U. Kühnel, M. Kübel, M. F. Kling, A. Yamada, 
K. Motomura, K. Ueda, R. Treusch, C. D. Schröter, R. Moshammer, and J. Ullrich, in Multiphoton Processes and Attosecond Physics, edited by K. Yamanouchi and K. Midorikawa (Springer, Heidelberg, 2012), p. 61.

[63] R. Guichard, M. Richter, J-M. Rost, U. Saalmann, A. A. Sorokin, and K. Tiedtke, J. Phys. B 46, 164025 (2013).

[64] F. Sorgenfrei, W. F. Schlotter, T. Beeck, M. Nagasono, S. Gieschen, H. Meyer, A. Föhlisch, M. Beye, and W. Wurth, Rev. Sci. Instrum. 81, 043107 (2010).

[65] M. Wöstmann, R. Mitzner, T. Noll, S. Roling, B. Siemer, F. Siewert, S. Eppenhoff, F. Wahlert, and H. Zacharias, J. Phys. B 46, 164005 (2013).

[66] C. Gahl, A. Azima, M. Beye, M. Deppe, K. Döbrich, U. Hasslinger, F. Hennies, A. Melnikov, M. Nagasono, A. Pietzsch, M. Wolf, W. Wurth, and A. Föhlisch, Nat. Photonics 2, 165 (2008).

[67] O. Krupin, M. Trigo, W. F. Schlotter, M. Beye, F. Sorgenfrei, J. J. Turner, D. A. Reis, N. Gerken, S. Lee, W. S. Lee, G. Hays, Y. Acremann, B. Abbey, R. Coffee, M. Messerschmidt, S. P. Hau-Riege, G. Lapertot, J. Lüning, P. Heimann, R. Soufli, M. Fernandez-Perea, M. Rowen, M. Holmes, S. L. Molodtsov, A. Föhlisch, and W. Wurth, Opt. Express 20, 11396 (2012).

[68] M. Beye, O. Krupin, G. Hays, A. H. Reid, D. Rupp, S. de Jong, S. Lee, W.-S. Lee, Y.-D. Chuang, R. Coffee, J. P. Cryan, J. M. Glownia, A. Föhlisch, M. R. Holmes, A. R. Fry, W. E. White, C. Bostedt, A. O. Scherz, H. A. Duerr, and W. F. Schlotter, Appl. Phys. Lett. 100, 121108 (2012).

[69] M. Drescher, M. Hentschel, R. Kienberger, G. Tempea, C. Spielmann, G. A. Reider, P. B. Corkum, and F. Krausz, Science 291, 1923 (2001).

[70] M. Uiberacker, E. Goulielmakis, R. Kienberger, A. Baltuska, T. Westerwalbesloh, U. Keineberg, U. Heinzmann, M. Drescher, and F. Krausz, Laser Phys. 15, 195 (2005).

[71] E. Goulielmakis et al., Science 305, 1267 (2004).

[72] F. Krausz and M. Ivanov, Rev. Mod. Phys. 81, 163 (2009).

[73] U. Frühling, J. Phys. B 44, 243001 (2011).

[74] B. Schütte, U. Frühling, M. Wieland, A. Azima, and M. Drescher, Opt. Express 19, 18833 (2011).

[75] O. Grimm, K. Klose, and S. Schreiber, in Proceedings of the 10th European Particle Accelerator Conference,
Edinburgh, Scotland, 2006 (EPS-AG, Edinburgh, Scotland, 2006), p. 3143.

[76] K. Flöttmann, ASTRA user manual, http://www.desy.de/ $\sim$ mpyflo/Astra_dokumentation/.

[77] M. Dohlus and T. Limberg, in Proceedings of FEL2004 Conference (Comitato Conferenze Elettra, Trieste, Italy, 2004), MOCOS05.

[78] S. Reiche, Nucl. Instrum. Methods Phys. Res., Sect. A 429, 243 (1999).

[79] M. Minty and F. Zimmermann, Measurement and Control of Charged Particle Beams (Springer, New York, 2003).

[80] M. Sands, Report No. SLAC-AP-085, 1991.

[81] N. Gerken, S. Klumpp, A. A. Sorokin, K. Tiedtke, M. Richter, V. Bürk, K. Mertens, P. Juranic, and M. Martins, Phys. Rev. Lett. 112, 213002 (2014).

[82] T. Maltezopoulos, S. Cunovic, M. Wieland, M. Beye, A. Azima, H. Redlin, M. Krikunova, R. Kalms, U. Fruehling, F. Budzyn, W. Wurth, A. Foehlisch, and M. Drescher, New J. Phys. 10, 033026 (2008).

[83] E. Allaria et al., Nat. Photonics 6, 699 (2012).

[84] B. Faatz, N. Baboi, V. Ayvazyan, V. Balandin, W. Decking, S. Düsterer, H.-J. Eckoldt, J. Feldhaus, N. Golubeva, K. Honkavaara, M. Koerfer, T. Laarmann, A. Leuschner, L. Lilje, T. Limberg, D. Noelle, F. Obier, A. Petrov, E. Ploenjes, K. Rehlich, H. Schlarb, B. Schmidt, M. Schmitz, S. Schreiber, H. Schulte-Schrepping, J. Spengler, M. Staack, F. Tavella, K. Tiedtke, M. Tischer, R. Treusch, M. Vogt, A. Willner, J. Bahrdt, R. Follath, M. Gensch, K. Holldack, A. Meseck, R. Mitzner, M. Drescher, V. Miltchev, J. Rönsch-Schulenburg, and J. Rossbach, Nucl. Instrum. Methods Phys. Res., Sect. A 635, S2 (2011).

[85] S. Ackermann, A. Azima, S. Bajt, J. Boedewadt, F. Curbis, H. Dachraoui, H. Delsim-Hashemi, M. Drescher, S. Duesterer, B. Faatz, M. Felber, J. Feldhaus, E. Hass, U. Hipp, K. Honkavaara, R. Ischebeck, S. Khan, T. Laarmann, C. Lechner, Th. Maltezopoulos, V. Miltchev, M. Mittenzwey, M. Rehders, J. Rönsch-Schulenburg, J. Rossbach, H. Schlarb, S. Schreiber, L. Schroedter, M. Schulz, S. Schulz, R. Tarkeshian, M. Tischer, V. Wacker, and M. Wieland, Phys. Rev. Lett. 111, 114801 (2013). 